\title{
Bridging the Gap: The Importance of TUBA1A $\alpha$-Tubulin in Forming Midline Commissures
}

\author{
Georgia Buscaglia ${ }^{1 \dagger}$, Kyle R. Northington ${ }^{1+}$, Jayne Aiken ${ }^{1,2}$, Katelyn J. Hoff ${ }^{2}$ and \\ Emily A. Bates ${ }^{1 *}$
}

${ }^{1}$ Department of Pediatrics, University of Colorado Anschutz Medical Campus, Aurora, CO, United States, ${ }^{2}$ Department of Cell and Developmental Biology, University of Colorado Anschutz Medical Campus, Aurora, CO, United States

\section{OPEN ACCESS}

Edited by:

Richard Luduena, The University of Texas Health Science Center at San Antonio, United States

Reviewed by:

Monika S. Brill,

Technical University of Munich,

Germany

Emmanuel Derivery,

University of Cambridge,

United Kingdom

Pavel Draber,

Institute of Molecular Genetics

(ASCR), Czechia

*Correspondence:

Emily A. Bates

Emily.Bates@CUAnschutz.edu

${ }^{t}$ These authors have contributed equally to this work

Specialty section:

This article was submitted to

Cellular Biochemistry,

a section of the journal

Frontiers in Cell and Developmental

Biology

Received: 04 October 2021

Accepted: 20 December 2021

Published: 19 January 2022

Citation:

Buscaglia G, Northington KR, Aiken J, Hoff KJ and Bates EA (2022) Bridging the Gap: The Importance of TUBA1A $\alpha$-Tubulin in Forming

Midline Commissures.

Front. Cell Dev. Biol. 9:789438. doi: 10.3389/fcell.2021.789438
Developing neurons undergo dramatic morphological changes to appropriately migrate and extend axons to make synaptic connections. The microtubule cytoskeleton, made of $\alpha / \beta$-tubulin dimers, drives neurite outgrowth, promotes neuronal growth cone responses, and facilitates intracellular transport of critical cargoes during neurodevelopment. TUBA1A constitutes the majority of $\alpha$-tubulin in the developing brain and mutations to TUBA $1 A$ in humans cause severe brain malformations accompanied by varying neurological defects, collectively termed tubulinopathies. Studies of TUBA1A function in mammalian cells have been limited by the presence of multiple genes encoding highly similar tubulin proteins, which leads to $\alpha$-tubulin antibody promiscuity and makes genetic manipulation challenging. Here, we test mutant tubulin levels and assembly activity and analyze the impact of TUBA1A reduction on growth cone composition, neurite extension, and commissural axon architecture during brain development. We present a novel tagging method for studying and manipulating TUBA1A in cells without impairing tubulin function. Using this tool, we show that a TUBA1A loss-of-function mutation TUBA1A N102D $\left(T U B A 1 A^{N D}\right)$, reduces TUBA1A protein levels and prevents incorporation of TUBA1A into microtubule polymers. Reduced Tuba1a a-tubulin in heterozygous Tuba1 $a^{\mathrm{ND/+}}$ mice leads to grossly normal brain formation except a significant impact on axon extension and impaired formation of forebrain commissures. Neurons with reduced Tuba1a as a result of the Tuba1 $a^{N D}$ mutation exhibit slower neuron outgrowth compared to controls. Neurons deficient in Tuba1a failed to localize microtubule associated protein-1b (Map1b) to the developing growth cone, likely impacting stabilization of microtubules. Overall, we show that reduced Tuba1a is sufficient to support neuronal migration and cortex development but not commissure formation, and provide mechanistic insight as to how TUBA1A tunes microtubule function to support neurodevelopment.

Keywords: TUBA1A protein, microtubule cytoskeleton, MAP1B, commissure defects, tubulinopathy

\section{INTRODUCTION}

Mammalian brain development is a complex process that requires precise coordination of multiple cell types and extracellular cues to form a fully specified tissue. Despite many advances in understanding the cellular and molecular players involved in brain development, there is still much that remains unknown. Insights into the molecular pathways governing neurodevelopment 
can be gained from studying genetic mutations that impair specific aspects of brain development. Severe cortical and neurodevelopmental phenotypes associated with mutations that disrupt tubulin genes, termed tubulinopathies, have recently been described in humans (Poirier et al., 2007; FalletBianco et al., 2008; Cushion et al., 2013; Oegema et al., 2015). Tubulinopathy mutations cause a spectrum of neurodevelopmental phenotypes, but frequently involve cortical malformations such as lissencephaly, agenesis or hypoplasia of the corpus callosum, and cerebellar hypoplasia (Poirier et al., 2007; Tischfield et al., 2011; Cushion et al., 2013; Oegema et al., 2015). Recent studies of human tubulinopathy mutations have revealed that each variant may impact different aspects of microtubule function, such as protein folding, polymerization competency, and microtubule-associated protein (MAP)-binding (Tian et al., 2008; Tian et al., 2010; Aiken et al., 2019a; Aiken et al., 2019b). Tubulin mutations can therefore be used to interrogate the requirement for specific aspects of microtubule function throughout neurodevelopment.

Developing neurons must migrate to the correct location, extend axons to meet sometimes distant synaptic partners, and form functional connections. Throughout this process, neurons undergo dramatic morphological changes that require coordinated interaction between the cytoskeleton and the extracellular environment. In post-mitotic neurons, microtubule polymers made of $\alpha / \beta$-tubulin dimers facilitate nucleokinesis and cellular migration, support growth cone navigation, promote axon formation and form the tracks upon which intracellular trafficking occurs (Sakakibara et al., 2013; Liu and Dwyer, 2014; Kapitein and Hoogenraad, 2015; Miller and Suter, 2018). The microtubule network needs to be precisely controlled to fulfill diverse functions in neurons. Microtubule properties can be modulated through post-translational modifications (PTMs) to tubulin subunits, association with MAPs, and through the particular tubulin genes, or isotypes, that a cell expresses (Gadadhar et al., 2017). The human genome contains at least nine unique $\alpha$ - and ten unique $\beta$-tubulin genes (Khodiyar et al., 2007; Findeisen et al., 2014). The $\alpha$-tubulin isotype encoded by the gene TUBA1A is abundant in the brain and is the most highly expressed a-tubulin in post-mitotic developing neurons (Miller et al., 1987; Gloster et al., 1994; Gloster et al., 1999). TUBA1A mutations are highly represented in cases of human tubulinopathies (Aiken et al., 2017), suggesting that TUBA1A plays an important role in neurodevelopment. However, the high degree of sequence conservation between a-tubulin genes has historically made studying TUBA1A function in cells challenging, due to the limited availability of tools.

Mouse models harboring mutations to Tubala can be used as tools to interrogate the function of Tubala in the context of the neuronal milieu. As tubulin genes are often required for life and the nucleotide sequence between isotypes is conserved, generation of mutant mouse lines to study Tubala function in vivo has been challenging. To date, only a handful of Tubala mutant mouse lines have been generated, three by ENU-induced forward genetic screens and one by site-directed CRISPR gene editing (Keays et al., 2007; Gartz Hanson et al., 2016; Bittermann et al., 2019; Leca et al., 2020). We previously revealed that the ENU-induced Tubala $a^{N 102 D}$ allele $\left(T u b a 1 a^{N D}\right)$ impairs microtubule function in both $S$. cerevisiae and mice through a loss-of-function mechanism by reducing $\alpha$-tubulin protein (Gartz Hanson et al., 2016; Buscaglia, 2020). Homozygous Tuba1a ${ }^{N D}$ mice exhibit severely impaired brain development and are neonatal lethal, similar to phenotypes seen in the Tuba1a null and Tuba1a-R215* mutant mice (Gartz Hanson et al., 2016; Aiken et al., 2017; Bittermann et al., 2019). In homozygous Tuba1 $a^{N D}$, Tuba1a ${ }^{\text {Null }}$ and Tuba1a-R215* mice, as well as many patients with TUBA1A-associated tubulinopathies, cortical migration and commissural formation are severely disrupted. This makes it difficult to infer whether axon pathfinding deficits are a direct consequence of altered Tubala function or if they are secondary to abnormal cortical layering and migration. Tubal $a^{N D /+}$ heterozygous mutant mice have reduced Tubala function during brain development, which is sufficient to support neuron survival and cortical layer formation (Gartz Hanson et al., 2016; Buscaglia, 2020), but does not support formation of axon commissures. Therefore, Tubala $a^{N D /+}$ heterozygous animals can provide insight into how Tubala contributes specifically to axon pathfinding.

Here, we show that a reduction in developmental Tubala protein impairs formation of large brain commissures. Using a novel tubulin visualization technique, we demonstrate that the TUBA $1 A^{N 102 D}$ mutation prevents incorporation of TUBA1A into microtubule polymers in cells. In mice, heterozygous $T u b a 1 a^{N D /+}$ brains fail to form the corpus callosum, anterior and hippocampal commissures. Cultured neurons from $T u b a 1 a^{N D /+}$ and wild-type cortices reveal that Tubala $a^{N D /+}$ neurons have shorter neurites than wild-type. Further, we demonstrate that Tubala $a^{N D /+}$ neurons fail to localize Map1b, a critical developmental MAP, to the developing growth cone. Neither Map1b expression nor binding to microtubules is impaired by reduced Tubala function. Trafficking along neurites is perturbed by reduced Tubala. Thus failure to localize Map1b is likely due to defects in trafficking. Collectively, our data present evidence that reduction of functional Tuba1a protein allows for normal neuron migration and cortical layering but prevents formation of commissures by impairing axon outgrowth.

\section{MATERIALS AND METHODS}

\section{Animals}

All animal research was performed in accordance with the Institutional Animal Care and Use Committee at the University of Colorado School of Medicine. All mice used were maintained on a 129S1/C57Bl6 genetic background. Mice were kept on a 12:12 light:dark cycle with ad libitum access to food and water. Tubala $a^{N D /+}$ mice were identified in an ENU mutagenesis screen that was conducted using C57Bl/6J mice and then outcrossed onto 129S1/Svlmj (Gartz Hanson et al., 2016). All of the data for this manuscript were obtained from mice that have been outcrossed over 20 generations to 129 S1. Tubala $a^{N D /+}$ and wild-type littermate mice were maintained on water 
supplemented with $0.2 \mathrm{~g} / \mathrm{L} \mathrm{MgSO}_{4}$ to promote Tubala $\mathrm{MD}^{\mathrm{N} /+}$ survival and ability to reproduce. Survival rates of Tuba1 $a^{N D /+}$ with $0.2 \mathrm{~g} / \mathrm{L}^{\mathrm{MgSO}_{4}}$ are $34 / 35$ total and without $\mathrm{MgSO}_{4}$ supplemented water are three out of nine pups. In contrast, 9/ 10 wild type litter mates survived without $\mathrm{MgSO}_{4}$ supplemented water and 47/47 wildtype littermates survive with $\mathrm{MgSO}_{4}$ supplemented water. For timed matings, male and female mice (Tuba1 $a^{N D /+} \mathrm{x}$ wild type) were introduced overnight and separated upon confirmation of mating, which was considered embryonic day 0.5. Male and female mice were represented in all studies. All mice were genotyped by PCR amplification of DNA isolated from tail snips followed by Sanger sequencing to differentiate homozygous or heterozygous Tubala $\mathrm{ND}^{\mathrm{ND}+}$ mice from wild-type. Primers used to amplify mouse DNA for genotyping were: forward:TGGATGGTACGCTTGGTCTT; reverse: CTTTGCAGATGAAGTTCGCA; and sequencing: GTCGAGGTTTCTACGACAGATATC.

\section{Histology}

Mice were anesthetized and trans-cardially perfused with $0.1 \mathrm{M}$ $\mathrm{NaCl}$ and $4 \%$ paraformaldehyde (PFA) for histology. Tissues of interest were dissected and post-fixed in 4\% PFA. Tissue sectioning was performed on a CM1520 cryostat (Leica, Wetzlar, Germany) and $30 \mu \mathrm{m}$ cryosections were obtained for all histology. For luxol fast blue staining, sections from brain were stained using a $0.1 \%$ luxol fast blue solution. For most immunofluorescence studies PFA-fixed tissues or cells were blocked in phosphate-buffered saline (PBS) containing 5\% goat serum or bovine serum albumin (BSA) with $0.3 \%$ Triton$\mathrm{X} 100$. Primary and secondary antibodies were diluted in PBS containing $1 \%$ BSA with $0.1 \%$ Triton-X 100.

\section{Electron Microscopy}

Mice used for electron microscopy were perfused with $0.1 \mathrm{M}$ $\mathrm{NaCl}$ and $2.5 \%$ glutaraldehyde $4 \%$ PFA, after which the brain was dissected and post-fixed in $2.5 \%$ glutaraldehyde $4 \%$ PFA overnight at $4^{\circ} \mathrm{C}$. Following post-fixation, brains were sent for sectioning and imaging by the CU School of Medicine Electron Microscopy Core facility. For analysis, Axons were counted in EM images only if they were captured completely in cross-section (round) and contained either a dark myelin ring surrounding, or had features of an axon (intracellular space had uniform electron density). Structures were only considered as compacted myelin sheaths if they were surrounding a structure resembling a crosssectional axon and were electron dense.

\section{Plasmids and Reagents}

The hexahisitidine (His6) epitope tag was inserted in the atubulin internal loop region (Schatz et al., 1987; Heilemann et al., 2008; Hotta et al., 2016). Codon optimization for rattus norvegicus (https://www.idtdna.com/codonopt) was used to generate the His6 sequence CATCACCACCATCATCAC, which was inserted into the coding region of human TUBA1A from the Mammalian Genome Collection (clone ID: LIFESEQ6302156) between residues I42 and G43. Gibson cloning was used to insert the gBlock of TUBA1A internally tagged with His6 (TUBA1A-His6) into the pCIG2 plasmid (shared by Dr. Matthew Kennedy, University of Colorado) linearized with NruI and HindIII. TUBA1A-His6 incorporation was confirmed by sequencing across the complete TUBA1A coding region. The TUBA1A ${ }^{T 349 E}$ $\left(T U B A 1 A^{T E}\right)$ polymerization incompetent, and TUBA1A ${ }^{E 255 A}$ $\left(T U B A 1 A^{E A}\right)$ highly polymer-stable a-tubulin mutants were identified and described in prior publications (Anders and Botstein, 2001; Johnson et al., 2011; Roostalu et al., 2020). To generate the GFP-TUBA1A vector, the coding region of human TUBA1A from the Mammalian Genome Collection (clone ID: LIFESEQ6302156) was amplified with forward primer TATGGC GGCCGCAGAGTGCTGGTAGTGCTGGTAGTGCTGGTATGC GTGAGTGCATCTCC and reverse primer TATGGCGGCCGCTTA GTATTCCTCTCCTTCTTCCTCACC. The resulting amplicon encompasses the BsrGI cutsite present at the end of GFP, a linker sequence (tripeptide linker SAG), the TUBA1A coding region, and terminates in a NotI site. This amplicon was cloned into the pCIG2 vector at the end of the GFP sequence using sticky-end cloning with BsrGI and NotI. The GFP-MACF43 vector was shared by Dr. Laura Anne Lowery (Boston College) and Dr. Casper Hoogenraad (Utrecht University). Myr-TdTomato plasmid DNA was shared from Dr. Mark Gutierrez and Dr. Santos Franco (University of Colorado).

\section{Cell Culture and Nucleofection}

Cos-7 cells (Thermo Fisher Scientific, Waltham, MA; ATCC ${ }^{\circledR}$ CRL-1651 ${ }^{\mathrm{TM}}$ ) were cultured in a $37^{\circ} \mathrm{C}$ humidified incubator with $5 \% \mathrm{CO}_{2}$ in DMEM (Gibco) supplemented with $10 \%$ fetal bovine serum (Gibco), $1 \mathrm{mM}$ sodium pyruvate (Thermo), and penicillin/ streptomycin $(1,000 \mathrm{IU} / 1,000 \mu \mathrm{g} / \mathrm{ml}$; Thermo). Cos-7 cells were transfected with $2.5 \mu \mathrm{g}$ of hexahistidine (His6) tagged TUBA1A plasmid DNA using Lipofectamine 3000 (Invitrogen). The Cos-7 cells were fixed and imaged $24 \mathrm{~h}$ after the addition of the TUBA $1 A$ plasmid. Cells were washed with PBS and PHEM buffer $(60 \mathrm{mM}$ PIPES, $25 \mathrm{mM}$ HEPES, $10 \mathrm{mM}$ EGTA, $2 \mathrm{mM} \mathrm{Mg} 2 \mathrm{Cl}$ ), and then fixed in $2 \%$ formaldehyde plus $0.05 \%$ glutaraldehyde. Immunostaining was performed using primary antibodies directed against: 6X-Histidine (Invitrogen, 4A12E4 37-2900; 1: 500), Acetylated Tubulin (Cell Signaling Technology, D20G3; 1: $800)$. Primary antibodies were diluted in blocking buffer (5\% BSA with $0.3 \%$ Triton-X 100 in PBS) and incubated overnight at $4^{\circ} \mathrm{C}$ in a humidified chamber. After primary antibody staining, cells were washed three times with PBS. Fluorescently-conjugated secondary antibodies were diluted $1: 500$ in $1 \%$ BSA with $0.1 \%$ Triton-X 100 in PBS and incubated for $1 \mathrm{~h}$ at room temperature, protected from light. Secondary antibodies were from Life Technologies (Carlsbad, CA) all used at 1:500. For Cos-7 proteasome inhibition assays, $5 \mu \mathrm{M}$ Lactacystin A (Tomoda and Omura, 2000; Ōmura and Crump, 2019) was added to normal culture medium for $24 \mathrm{~h}$, the day following transfection with TUBA1A-His6 constructs. Dissociated neurons were cultured from male and female P0-P2 mouse or rat cortices. Brains were removed and placed into Hanks Balanced Salt Solution (HBSS, Life Technologies) supplemented with $9.9 \mathrm{mM}$ HEPES (Life Technologies) and $1 \mathrm{mM}$ kynurenic acid (Tocris Bioscience, Bristol, United Kingdom). Meninges were removed and cortices were dissected and cut into approximately $1 \mathrm{~mm}$ pieces. Cortical pieces 
were triturated to a single-cell suspension using glass Pasteur pipettes. Cortical neurons were plated onto $35 \mathrm{~mm}$ Poly-DLysine coated glass-bottom culture dishes at a density of 350,000 cells (Willco Wells, HBSt-3522). For nucelofected mouse and rat neurons, $4 \mu \mathrm{g}$ of plasmid DNA was introduced to $4 \times 10^{6}$ neurons using an AMAXA nucleofection kit (VPG1001, VPG-1003; Lonza). AMAXA-nucleofected cells were plated in $35 \mathrm{~mm}$ glass bottom imaging dishes. Neurons were maintained in a $37^{\circ} \mathrm{C}$ humidified incubator with $5 \% \mathrm{CO}_{2}$ in phenol-free Neurobasal-A medium (Life Technologies) supplemented with B-27 (Thermo Fisher Scientific, Waltham, MA), Penn/strep (Thermo), GlutaMax (Thermo), $5 \mathrm{ng} / \mathrm{ml} \beta$-FGF (Gibco), and Sodium Pyruvate (Thermo).

\section{RNA Isolation + RTPCR}

RNA was isolated from Cos-7 cells, $48 \mathrm{~h}$ post-transfection using TRIzol Reagent (Thermo; 15596026). RNA concentration and purity were determined using a spectrophotometer, then cDNA was synthesized using the RT2 First Strand Kit (Qiagen, Hilden, Germany; 330401). qRT-PCR reactions were prepared with SYBR Green RT-PCR Master mix (Thermo; S9194) and run with a CFX Connect Real-Time System (Bio-Rad). Samples were run in triplicate, results were analyzed in Excel. All qPCR data presented in this manuscript was normalized to expression of GFP, which was present on the same plasmid as TUBA1A-His6 constructs. Wild-type TUBA1A mRNA quantity was set to $=1$ and $T U B A 1 A^{N D}$ relative mRNA quantity was presented relative to wild-type. For all qRT-PCR experiments 3 biological replicates were used per genotype.

\section{Neuron Immunocytochemistry}

DIV 2 primary cortical neurons were washed with PBS and fixed with a fixation solution of $4 \%$ PFA and $0.2 \%$ glutaraldehyde in PBS for $15 \mathrm{~min}$ at room temperature. For tubulin extraction, cells were washed with PBS followed by PHEM buffer (60 mM PIPES, $25 \mathrm{mM}$ HEPES, $10 \mathrm{mM}$ EGTA, $2 \mathrm{mM} \mathrm{Mg} 2 \mathrm{Cl}$ ) then soluble tubulin dimers were extracted using $0.1 \%$ triton with $10 \mu \mathrm{M}$ taxol and 0.1\% DMSO in PHEM buffer. Extracted cells were fixed with $2 \%$ PFA and $0.05 \%$ glutaraldehyde in PBS for $10 \mathrm{~min}$, washed with PBS and then reduced in $0.1 \% \mathrm{NaBH} 4$ in PBS for 7 min at room temperature. Cells were then washed with PBS and blocked in 3\% BSA and $0.2 \%$ Triton in PBS for $20 \mathrm{~min}$ at room temperature, with agitation. Immunostaining was performed using primary antibodies directed against: 6X-Histidine (Invitrogen, 4A12E4 37-2900; 1:500), total a-tubulin (Sigma, DM1A T6199; 1:5,000), Acetylated Tubulin (Sigma, T7451; 1: 1,000), Tyrosinated Tubulin (Chemicon, MAB 1864; 1:1,000), Map1b (Santa Cruz Biotech, sc-135978; 1:500), Map2 (Novus Biologicals, NB300-213; 1:2,000). Primary antibodies were diluted in blocking buffer (5\% BSA with $0.3 \%$ Triton-X 100 in PBS) and incubated overnight at $4^{\circ} \mathrm{C}$ in a humidified chamber. After primary antibody staining, cells were washed three times with PBS. Fluorescently-conjugated secondary antibodies were diluted 1:500 in 1\% BSA with $0.1 \%$ Triton-X 100 in PBS and incubated for $2 \mathrm{~h}$ at room temperature, protected from light. Secondary antibodies were from Life Technologies (Carlsbad, CA) all used at 1:500. Alexa Fluor 568-conjugated Phalloidin
(Thermo, A12380; 1:20) was added during secondary antibody incubation for labeling of filamentous actin. Tissues or cells of interest were mounted onto glass microscope slides and sealed with glass coverslips and aqueous mounting media containing DAPI (Vector Laboratories, \#H-1200) and imaged on a Zeiss 780 or 880 confocal microscope with a $\times 40$ or $\times 63$ oil objective.

\section{Western Blotting}

Protein was isolated from brains of P0-P2 mice by dounce homogenization and ultra-centrifugation at $100,000 \times \mathrm{g}$ for $45 \mathrm{~min}$ at $4^{\circ} \mathrm{C}$. Maplb was quantified in three different scenarios. Total Map1b was quantified from the whole brain lysates. For Map1b associated with tubulin, the tubulin-enriched fraction was isolated either with taxol at $37^{\circ} \mathrm{C}$ according to a previously established protocol (Vallee, 1982) or without taxol at $37^{\circ} \mathrm{C}$ using centrifugation to separate the tubulin-rich fraction. Cos-7 cell protein was extracted using a Tris-Triton lysis buffer with protease inhibitor cocktail (Sigma). Protein concentrations were assessed using a BCA assay (Thermo), and relative concentration was determined using a Synergy H1 microplate reader (BioTek Instruments, Winooski, VT). $5 \mu \mathrm{g}$ of either whole brain lysate or tubulin-enriched protein fraction was loaded per lane and run on 4-20\% Mini-Protean TGX Stain-Free precast gels (4568093; Bio-Rad Laboratories, Hercules, CA) at $150 \mathrm{mV}$ for $1 \mathrm{~h}$. Prior to protein transfer, Stain-Free gels were activated with UV light for $1 \mathrm{~min}$ and imaged for total protein on gel using a ChemiDoc MP imager (Bio-Rad). Proteins were transferred to PVDF blotting membranes (Bio-Rad) in standard $25 \mathrm{mM}$ Trisbase, $192 \mathrm{mM}$ glycine, 15\% methanol transfer buffer, or transfer buffer optimized for high molecular-weight proteins $(>200 \mathrm{kDa})$ by the addition of $0.025 \%$ SDS. Blots were transferred at $4^{\circ} \mathrm{C}$ and $75 \mathrm{~V}$ for either $1 \mathrm{~h}$ for standard molecular-weight proteins, or $3 \mathrm{~h}$ for high molecular-weight proteins. Immediately following transfer, PVDF membranes were rinsed in TBST and imaged to quantify the total protein on blot using UV-activated StainFree blots. Gels were also imaged post-transfer to assess transfer efficiency for each blot. Membranes were blocked in Tris-buffered Saline containing 0.1\% Tween-20 (TBST) with 5\% BSA for $1 \mathrm{~h}$ and incubated in primary antibody diluted in TBST containing $1 \%$ BSA overnight at $4^{\circ} \mathrm{C}$. Primary antibodies were: mouse anti 6X-Histidine (Invitrogen, 4A12E4; 1:500), chicken anti-GFP (Invitrogen, A10262; 1:1,000), and mouse anti-Maplb (Santa Cruz, sc-135978; 1:500). Blots were incubated in HRPconjugated Goat-anti-mouse (1:5,000; Santa Cruz) or goatanti-chicken (1:5,000; Santa Cruz) secondary antibody diluted in TBST containing $0.5 \%$ BSA with streptavidin-HRP (Bio-Rad, $1: 10,000)$ for band visualization for $1 \mathrm{~h}$ at room temperature. Blots were developed in ECL solution for $5 \mathrm{~min}$ at room temperature (Bio-Rad) and imaged.

\section{Netrin-1 Experiments}

Primary wild-type and Tuba1a $a^{N D /+}$ neonatal (P0-P3) mouse cortical neurons were nucleofected with $2 \mu \mathrm{g}$ of MyrTdTomato plasmid DNA and cultured as described above. Netrin-1 expressing plasmid (OriGene Cat\#: MG223704) was transfected into Cos-7 cells using Lipofectamine 3000 (Thermo Fisher Scientific Cat\#: L3000015). Cells were washed and media 
was replaced with Opti-MEM (Thermo Fisher Scientific Cat\#: 31985062). The following day media was removed from cells and placed in an equilibrated Amicon $30 \mathrm{kDa}$ molecular weight cutoff ultracentrifuge tube (Millipore Sigma Cat\#: UFC903008). The purified Netrin-1 was collected and quantified using a BCA assay (Thermo Fisher Scientific Cat \#:23227). Neurons were measured at DIV1 at $10 \mathrm{~min}$ intervals for $60 \mathrm{~min}$, Netrin- 1 was added at a final concentration of $500 \mathrm{ng} / \mathrm{ml}$ to the media and images were taken for $60 \mathrm{~min}$ after the addition of the chemoattractant. Neurons were measured using ImageJ/FIJI software (NIH) and Excel (Microsoft). Statistical analyses were performed, and graphs were created using Prism version 9.0 (GraphPad). For all statistical analyses, $p<0.05$ was considered statistically significant. Statistical analyses used in each experiment are indicated in their respective figure legends. T-tests were used for any comparisons between two groups and ANOVA was used for any comparison of more than two groups.

\section{dSTORM Super-Resolution Imaging and Analysis}

Primary neuronal cultures were generated and immunostained as described in the Cell Culture and Nucleofection and Neuron Immunocytochemistry sections, excepting the mounting step.

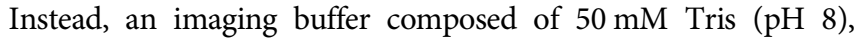
$10 \mathrm{mM} \mathrm{NaCl}, 10 \%$ glucose, $10 \mathrm{mM}$ mercaptoethylamin (MEA, Sigma-Aldrich, M6500), $0.56 \mathrm{mg} / \mathrm{ml}$ glucose oxidase (SigmaAldrich, G0543), and $34 \mu \mathrm{g} / \mathrm{ml}$ catalase (Sigma-Aldrich, C3155) was sterile filtered onto the sample. The cells were then imaged on a Ziess Elyra P.1 microscope using a Plan-Apochromat 63X 1.4NA objective to achieve high excitation power as previously described (Heilemann et al., 2008; Schüttpelz, 2010). In short, the Alexa 647labeled sample was excited into a high energy state where the majority of fluorophores enter a long-lived dark state while a small fraction of fluorophores are excited into a bright fluorescent state. Over the imaging period, the cycling of fluorophores from the ground state to either of the excited states, stochastically the dark or bright state, provides fluorophore position information. Superresolution images were generated by running the raw data through a series of analysis steps. First, the fluorescence background was removed using a previously described temporal median filter in Matlab (Matlab code provided by the University of Colorado School of Medicine Advanced Light Microscopy Core) (Hoogendoorn et al., 2014). Fluorophore positions were then fitted using the ThunderSTORM plugin for ImageJ and Fiji (Schneider et al., 2012; Ovesný, 2014). Sample drift was corrected using redundant cross-correlation algorithm in Matlab as previously described (Wang et al., 2014). Finally, a superresolution image was created by starting out with all pixels set to zero and incrementing the pixel value by one for every molecule localization that falls into that pixel.

\section{Experimental Design and Statistical Analyses}

Band volume of all Western blots was analyzed using Image Lab software (Bio-Rad). Fluorescence intensity measurements, area and morphological assessment, kymograph generation, and quantification of EM images was performed using ImageJ/FIJI software (NIH) and Excel (Microsoft). Statistical analyses were performed, and graphs were created using Prism version 8.0 (GraphPad). Most graphs display all data points to accurately represent the variability in each dataset, except in cases where such presentation obscured visibility. For all statistical analyses, statistical significance was considered to be $p<0.05$. Statistical analyses used in each experiment are indicated in their respective figure legends. For all graphs mean \pm SEM was reported unless otherwise noted. Normality of each dataset was assessed using a Shapiro-Wilk test. In datasets with two groups, parametric data was analyzed using a Student's t-test, while non-parametric data was assessed by Mann-Whitney $U$ analysis of medians. Multiple groups were compared by one-way or two-way ANOVA and analyzed post hoc by either a Bonferroni or Kruskal-Wallis test for parametric and non-parametric data, respectively.

\section{RESULTS}

\section{TUBA1A $^{N D} \alpha$-Tubulin does not Incorporate into Neuronal Microtubules}

The high degree of sequence similarity between a-tubulin isotypes has limited study of individual tubulin isotypes at the protein level in cells. As TUBA1A shares 99.5\% homology with TUBA1B a-tubulin (only 2 distinct amino acids), commercially available "TUBA1A" specific antibodies are promiscuous and bind more than the intended isotype target. Further, prior attempts to tag TUBA1A neuronal microtubules with $\mathrm{N}$ - or C-terminal fluorescent fusion proteins have had detrimental effects on protein function (Gonzalez-Garay and Cabral, 1996; Kimble et al., 2000). These challenges have made the direct visualization of specific tubulin isotypes or mutant tubulin proteins in neurons difficult. Thus, the ways in which TUBA1A specifically contributes to neuronal microtubule protein function have been difficult to ascertain. To address the need for better tools to study TUBA1A protein, we generated a functional hexahistidine (His6)-tagged TUBA1A construct based on a previously identified internal loop in the a-tubulin protein to aid visualization of TUBA1A in mammalian cells (Schatz et al., 1987) (Figure 1A). We inserted the His6 tag into an internal loop of TUBA1A between residues I42 and G43, a region of $\alpha$-tubulin that tolerates addition of amino acids without disrupting tubulin function (Schatz et al., 1987). Addition of the His6 tag in this loop has previously been used to affinity purify tubulin subunits for reconstituted systems (Heilemann et al., 2008; Hotta et al., 2016). However, to our knowledge this internal His6 tag on a-tubulin has never been used for immunohistochemical assays to visualize isotype-specific localization in cells.

Ectopically expressed wild-type TUBA1A-His6 is incorporated into Cos-7 cell microtubules and colocalizes with polymerized acetylated tubulin (Pearson coefficient $=0.31 \pm 0.03$, Figures $\left.\mathbf{1 B}, \mathbf{B}^{\prime}\right)$. Along with wild-type TUBA1A-His6, we ectopically expressed three TUBA1A variants in Cos-7 cells. Mutant TUBA1A-His6 ectopic expression in cells facilitates 


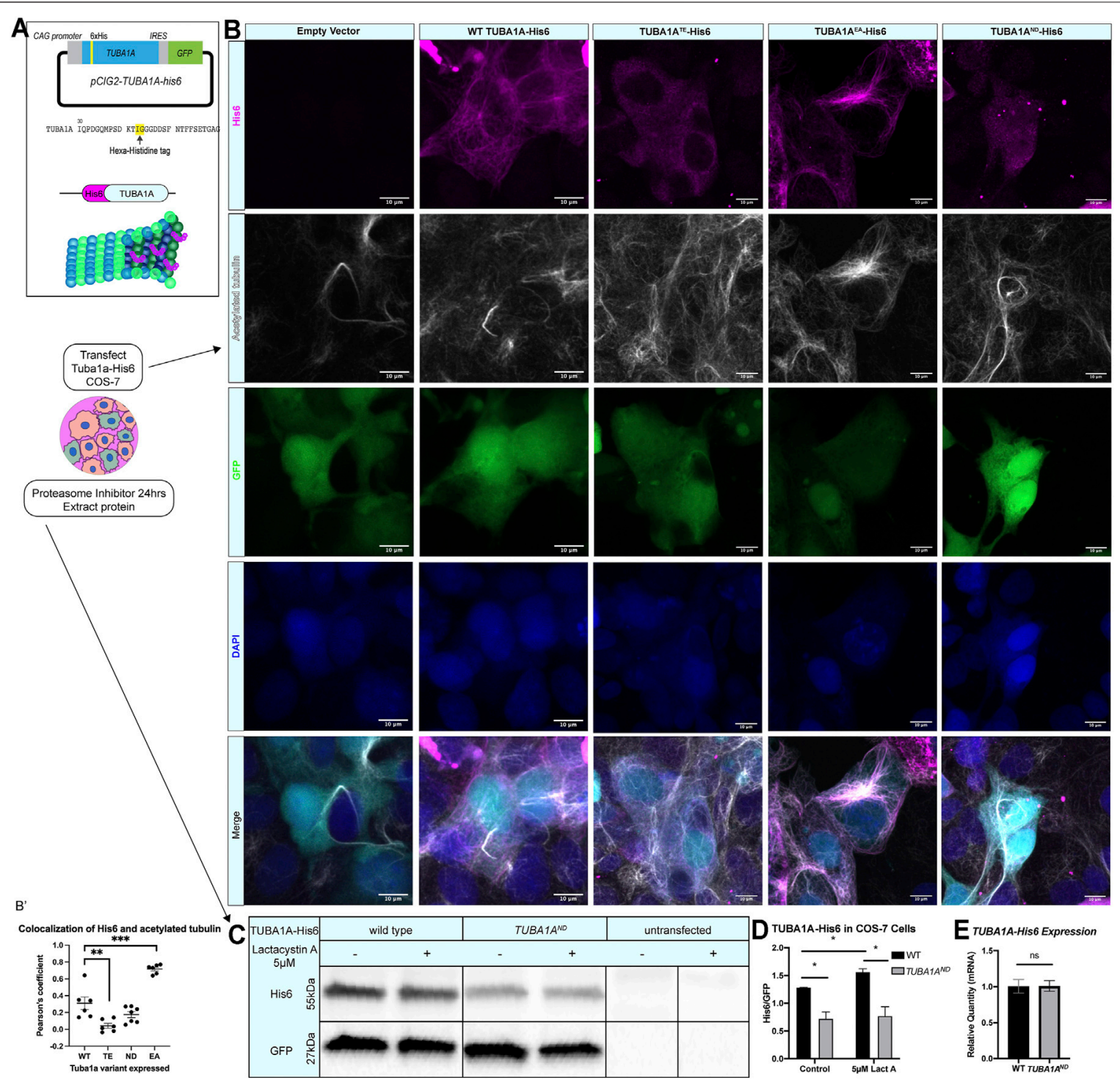

FIGURE 1 | TUBA1A $A^{N D}$ impairs incorporation into cellular microtubules and reduces $\boldsymbol{\alpha}$-tubulin protein abundance. (A) Schematic of TUBA1A-His6 tag and experimental design. His6 epitope tag was added to an internal loop on TUBA1A (top), and TUBA1A-His6 plasmid DNA was transfected into Cos-7 cells to label cellular microtubules in the presence or absence of proteasome inhibitor treatment (bottom). (B) Images showing Cos-7 cells transfected with an empty vector control, wild-type TUBA1A-His6, TUBA1A ${ }^{T E}$-His6 (polymerization-incompetent mutant), TUBA1A ${ }^{E A}$-His6 (highly polymer stable mutant), and TUBA1A ${ }^{N D}$-His6 plasmid DNA. Cells were immunolabeled with $\alpha$-His6 antibodies to reveal microtubule incorporation of wild-type and mutant TUBA1A-His6 protein. (B') Scatter plot showing the Pearsons' correlation coefficient to quantify the co-localization of acetylated tubulin with anti-His6 staining ${ }^{\star *} p<0.01$ and ${ }^{\star \star *} p<0.001$. (C) Western blot for His6 in TUBA1A-His6 transfected Cos-7 cell lysates. A subset of transfected cells were treated with $5 \mu \mathrm{M}$ Lactacystin A (LactA) for $24 \mathrm{~h}$ to block proteasomal degradation. His6 signal was normalized to GFP, which was expressed from the same plasmid. (D) Quantification of band density for His6 western blot shown in (C). His6 band density was normalized to GFP-expressing cells in control-treated (left), and cells treated with $5 \mu \mathrm{M}$ LactA for $24 \mathrm{~h}$ (right). Data were analyzed by $t$ test. $\mathrm{N}=3$ transfections; $n=3$ technical replicates; $p=0.04$ for all comparisons marked with asterisks; $p=0.83$ for control vs. LactA-treated TUBA1A ${ }^{N D}$. (E) Bar graph representing TUBA1A mRNA expression in Cos-7 cells transfected with TUBA1A-His6. TUBA1A mRNA expression was normalized to GFP mRNA expression. Data were normalized to TUBA1A expression in wild-type (WT) TUBA1A-His6-transfected cells and represent 3 separate transfection experiments and 3 qRT-PCR replicates. Differences between groups were assessed by $t$ test $(p=0.97)$. All images were taken at $\times 40$ magnification, scale bars are $10 \mu \mathrm{m}$. All graphs show mean of data \pm SEM, ${ }^{*} p<0.05$.

evaluation of the abundance and polymerization capability of mutant TUBA1A proteins. TUBA1A ${ }^{T 349 E}\left(T U B A 1 A^{T E}\right)$ is an $\alpha-$ tubulin mutation that prevents tubulin polymerization in yeast (Johnson et al., 2011) and acts as a negative control. The $T U B A 1 A^{T E}$-His6 shows low levels of punctate His6 staining that does not co-localize with microtubules consistent with previous findings that this mutation prevents $\mathrm{TUBA} 1 \mathrm{~A}^{\mathrm{TE}}$ incorporation into microtubules (Pearson coefficient $=0.042 \pm$ 0.07 , Figures $\left.\mathbf{1 B}, \mathbf{B}^{\prime}\right)$. However, acetylated tubulin is clearly visible in TUBA1A ${ }^{\mathrm{TE}}$ expressing cells indicating that exogenous expression of TUBA1A ${ }^{\mathrm{TE}}$ does not interfere with endogenous microtubule networks. In contrast, TUBA1 ${ }^{E 255 A}$ 

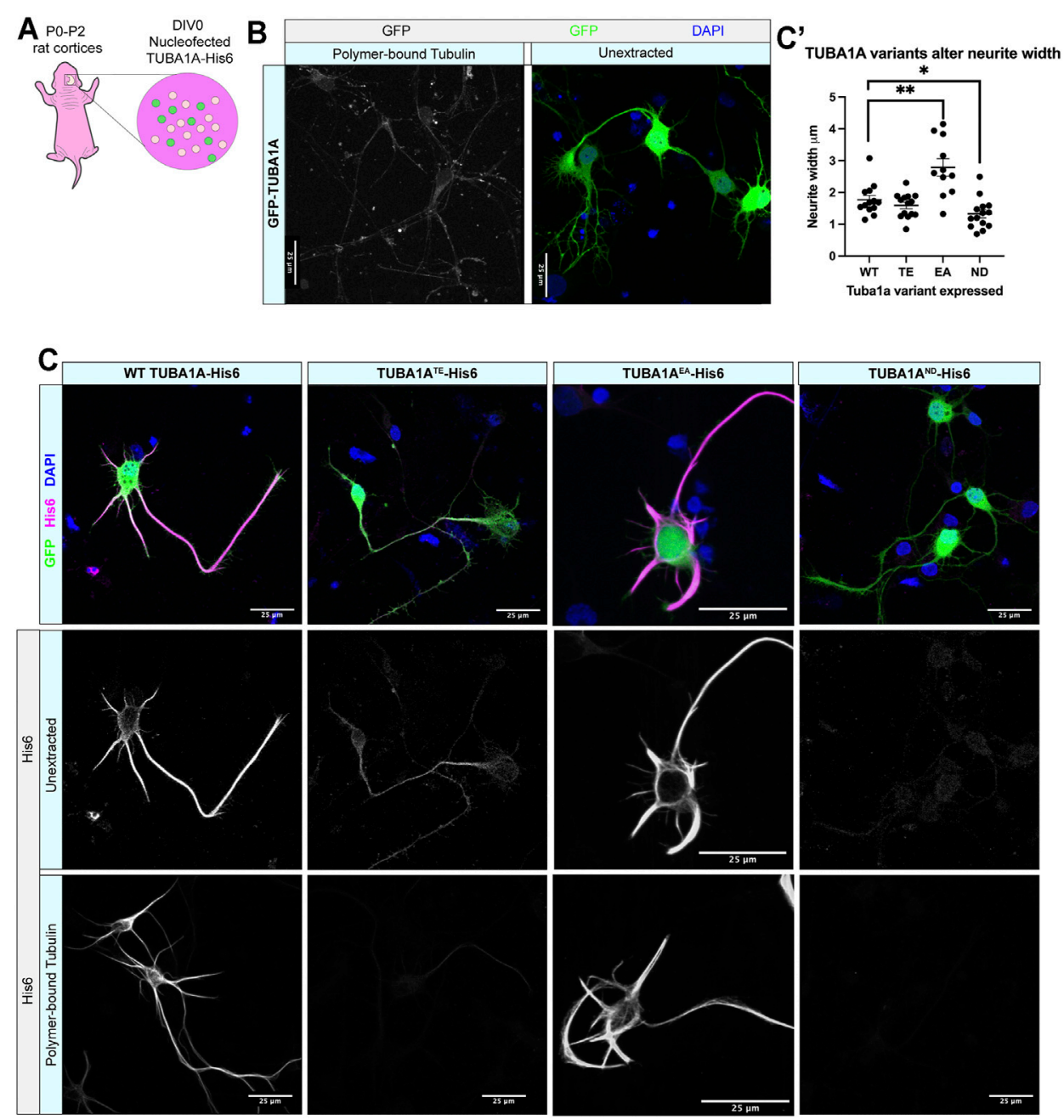

FIGURE 2 | TUBA1 $A^{N D} \alpha$-tubulin does not incorporate into neuronal microtubule polymers. (A) Schematic of cortical neuron isolation and transfection. (B) Cortical rat neurons at DIV 2 transfected with TUBA1A-GFP. Left panel shows neurons with soluble tubulin dimers extracted, showing only GFP-labeled TUBA1A that is incorporated into microtubule polymer. Right panel shows neurons with intracellular environment intact (unextracted), containing soluble tubulin dimers and polymerized microtubules transfected to express a TUBA1A-GFP fusion protein. (C) Rat cortical neurons at DIV 2 transfected with wild-type (WT) TUBA1A-His6 (far left), $T U B A 1 A^{T E}$-His6 polymerization-incompetent mutant as a negative control (middle left), TUBA1 $A^{E A}$ stabilizing mutant as a positive control (middle right), TUBA1 $A^{N D}$-His6 (far right). Top panels show composite image containing membrane-bound GFP (green) for confirmation of transfection, $a$-His6 (Magenta) and DAPI (blue) immunolabeling. Middle panels show unextracted and bottom panels show different DIV2 neurons after tubulin has been extracted to reveal only polymer-bound tubulin, labeled with $\alpha$-His6 antibodies to visualize ectopic TUBA1A-His6 proteins. Scale bar is $25 \mu \mathrm{m}$. (C') A Scatter plot shows that neurons expressing His6-Tuba1aEA have significantly wider neurites than neurons expressing His6-Tuba1aWT and neurons expressing His6-Tuba1aND have neurites that are significantly thinner than neurons expressing His6-Tuba1aWT ${ }^{*} p<0.05,{ }^{* *} p<0.01$.

(TUBA $1 A^{E A}$ ) is a microtubule stabilizing a-tubulin mutation which inhibits tubulin depolymerization and thus locks microtubules in a polymer-bound state (Anders and Botstein, 2001; Roostalu et al., 2020) (Figure 1B). The TUBA1A ${ }^{E A}$-His6 protein is abundantly incorporated into microtubule polymers (Pearson coefficient $0.72 \pm 0.02$, Figures $\left.\mathbf{1 B}, \mathbf{B}^{\prime}\right)$. Acetylated tubulin staining in TUBA1A ${ }^{E A}$-His6 is also increased compared to wild type. Previous reports demonstrate that the Tubala ${ }^{N D}$ variant causes partial loss of a-tubulin function in yeast and mice (Gartz Hanson et al., 2016; Buscaglia, 2020), but it was unclear whether or not the ND mutant tubulin is capable of polymerization. Our data reveal that TUBA1A ${ }^{\mathrm{ND}}$-His6 is not visible in microtubule polymers, but can be detected at low levels in the cytoplasm (Pearson coefficient $0.17 \pm 0.03$, Figures $\mathbf{1 B}, \mathbf{B}^{\prime}$ ). We conclude that $T U B A 1 A^{N D}$ does not incorporate into microtubules at detectable levels. The acetylated tubulin staining in TUBA1 $1 A^{N D}$-His6 cells is comparable to wild-type; microtubules are present, but TUBA1A ${ }^{\mathrm{ND}}$ protein is not incorporated.

Western blots of lysates from Cos-7 cells $48 \mathrm{~h}$ posttransfection reveal that TUBA $1 A^{N D}$-His6 protein is significantly reduced compared to wild-type TUBA1A-His6 (Figures 1C,D; $p=0.04$ ), despite similar TUBA1A mRNA levels between wild-type and TUBA1 ${ }^{N D}$ transfected Cos-7 
cells (Figure 1E; $p=0.97$ ). To evaluate if $T U B A 1 A^{N D}$ mutant protein is targeted for proteasomal degradation, we treated TUBA1A-His6 transfected Cos-7 cells with $5 \mu \mathrm{M}$ proteasome inhibitor, Lactacystin A [LactA (Tomoda and Omura, 2000; Ōmura and Crump, 2019)], for $24 \mathrm{~h}$ and probed for His6 abundance by western blot (Figure 1C). Treatment with LactA significantly increased wild-type TUBA1A-His6 protein compared to control-treated cells, but had no effect on $T U B A 1 A^{N D}$-His6 protein abundance (Figure 1D; $p=0.83$ ). These results indicate that $T U B A 1 A^{N D}$ mutant protein is likely not targeted for degradation by the proteasome. Overall, TUBA1A ${ }^{\mathrm{ND}}$ mutant protein is not incorporated into microtubules and is less abundant in the cells compared to wild type, despite similar mRNA levels.

We next investigated if $T U B A 1 A^{N D}$ substitution impairs incorporation of TUBA1A protein into neuronal microtubule polymers (Figure 2, Supplementary Figure S1). Wild-type rat cortical neurons were nucleofected with GFP-TUBA1A, wild-type TUBA1A-His6, TUBA1A ${ }^{T E}$-His6 (polymerization incompetent mutant), TUBA1A ${ }^{E A}$-His6 (stabilizing mutant), and TUBA $1 A^{N D}$-His6 DNA at day in vitro 0 (DIV 0; Figure 2A). Following 2 days in culture (DIV 2), cells were fixed and a subset of neurons were permeabilized to remove soluble tubulin dimers ("tubulin extraction"). Extraction of soluble tubulin leaves behind only polymer-bound tubulin, enabling visualization of ectopic tubulin protein polymerization competence (Zieve and Solomon, 1984). Neurons expressing GFP-TUBA1A exhibited abundant GFP signal in intact cells, but tubulin extraction revealed that GFP fusion impaired incorporation of TUBA1A into neuronal microtubule polymers (Figure 2B). In contrast, wild-type TUBA1A-His6 protein was abundantly visible in both unextracted and extracted neurons, demonstrating that His6tagging does not impair polymerization ability of TUBA1A in neurons (Figure 2C). As predicted, polymerization incompetent $T U B A 1 A^{T E}$-His6 mutant protein was diffusely visible in intact neurons, but was absent when only polymerized tubulin remained (after extraction) (Figure 2C, Supplementary Figure S1). The microtubule-stabilizing TUBA $1 A^{E A}-$ His6 was retained after extraction and incorporated into polymers more abundantly than wild-type, leading to stubby, microtubule-rich neurites (Figures 2C, $\mathbf{C}^{\prime}$ ). TUBA $1 A^{N D}$-His6 protein was detectable at very low levels in unextracted neurons, but was not visible following tubulin extraction, indicating that TUBA $1 A^{N D}$ impairs incorporation into neuronal microtubules (Figures $\mathbf{2 C}, \mathbf{C}^{\prime}$ ). These experiments suggest that $T U B A 1 A^{N D}$ reduces abundance of TUBA1A protein and prevents incorporation of mutant TUBA1A into cellular microtubules.

\section{Tuba1a is Required for Formation of Midline Commissures}

TUBA1A is a major component of developing neuronal microtubules and is critical for proper brain development (Aiken et al., 2017). Human TUBA1A tubulinopathy patients with heterozygous mutations in TUBA1A exhibit severe brain malformations including defects in commissure formation and changes to cortical folding patterns (lissencephaly, polymicrogyria, pachygyria). While homozygous Tuba1 $a^{N D / N D}$ mice have severe brain malformations including defects in cortical layer formation (Gartz Hanson et al., 2016), Tuba1a ${ }^{N D /+}$ heterozygous mutant mice undergo normal cortical migration, display comparable brain weight to wild-type littermates at birth, and survive to adulthood (Gartz Hanson et al., 2016; Buscaglia, 2020). However, 93\% (14/15 brains) have severe defects in corpus collosum formation including $87 \%(13 / 15)$ with complete agenesis of the corpus callosum and abnormal formation of the anterior and hippocampal commissures (Figure 3A). In wild-type mice, 12/12 had well-formed commissures. For a commissure to form properly, nascent callosal "pioneer" axons extend through midline at E15.5, and early "follower" axons begin extending at E17 in mice (Boyer and Gupton, 2018). Evidence of abnormal callosal projections were apparent as early as P0 in Tubala $a^{N D /+}$ brains, as seen by the early formation of aberrant axon bundles adjacent to the callosum, known as Probst bundles (Figure 3A) (Probst, 1901). In addition to agenesis of the corpus callosum at midline, lateral regions of adult Tuba1 $a^{N D /+}$ corpus callosum were found to be significantly thinner than wild-type (Figure 3B). Similarly, Tuba1 $a^{N D /+}$ anterior commissures are smaller than that of wildtype littermates (Figure 3C). In wild-type mice, corpus callosum thickness and anterior commissure area both increased significantly between P0 and adulthood; however, normal postnatal expansion of these tracts was not evident in Tuba1a ${ }^{N D /+}$ mice (Figures 3B,C). The corpus callosum is absent along the rostral-caudal axis in Tubala $a^{N D /+}$ animals. Tuba1 $a^{N D /+}$ axons fail to organize into typical midline commissural structures, indicating that half of the normal amount of Tubala during brain development is not sufficient for commissural formation (Buscaglia, 2020).

Examination of sagittal brain sections taken directly at midline revealed dramatic disorganization of corpus callosum axons in Tuba1 $a^{N D /+}$ brains (Figure 4A). Compared to wild-type, Tuba1 $a^{N D /+}$ midline commissural axons were largely absent, and the existing axons failed to organize into a tract with uniform orientation (Figure 4A). Despite dramatic differences between wild-type and Tuba1 $a^{N D /+}$ callosal axon organization, Tubala $a^{N D /+}$ axons were highly colocalized with immunolabeled myelin sheaths (Figure 4A). To further assess the impact of Tuba1 $a^{N D /+}$ substitution on callosal axon morphology and myelination, we performed electron microscopy (EM) in both wild-type and Tuba $1 a^{N D /+}$ corpus callosi. Due to the scarcity of axons present directly at midline in the Tuba1 $a^{N D /+}$ corpus callosum, we sampled a region of corpus callosum $2 \mathrm{~mm}$ lateral to midline for both wild-type and Tuba1 $a^{N D /+}$ animals (Figure 4B). EM images revealed a striking difference in axon density between wild-type and Tubal $a^{N D /+}$ corpus callosi (Figures 4B,C; $p=0.03$ ). Myelin thickness, measured by G-ratio, was similar between wild-type and Tuba $1 a^{N D /+}$ brains (Figure $4 D$; $p=0.34$ ), as was axon diameter (Figure 4E; $p=0.14$ ). There was a trend towards decreased myelination in Tuba1 $a^{N D /+}$ animals $(p=0.07)$, but this difference was not statistically significant (Figure 4F). These data provide evidence that Tuba $1 a^{N D /+}$ callosal axons do not correctly organize to form a commissure. Previously published data indicated that reduced developmental Tubala function is tolerable for cortical neuron migration (Gartz Hanson et al., 2016); however, 


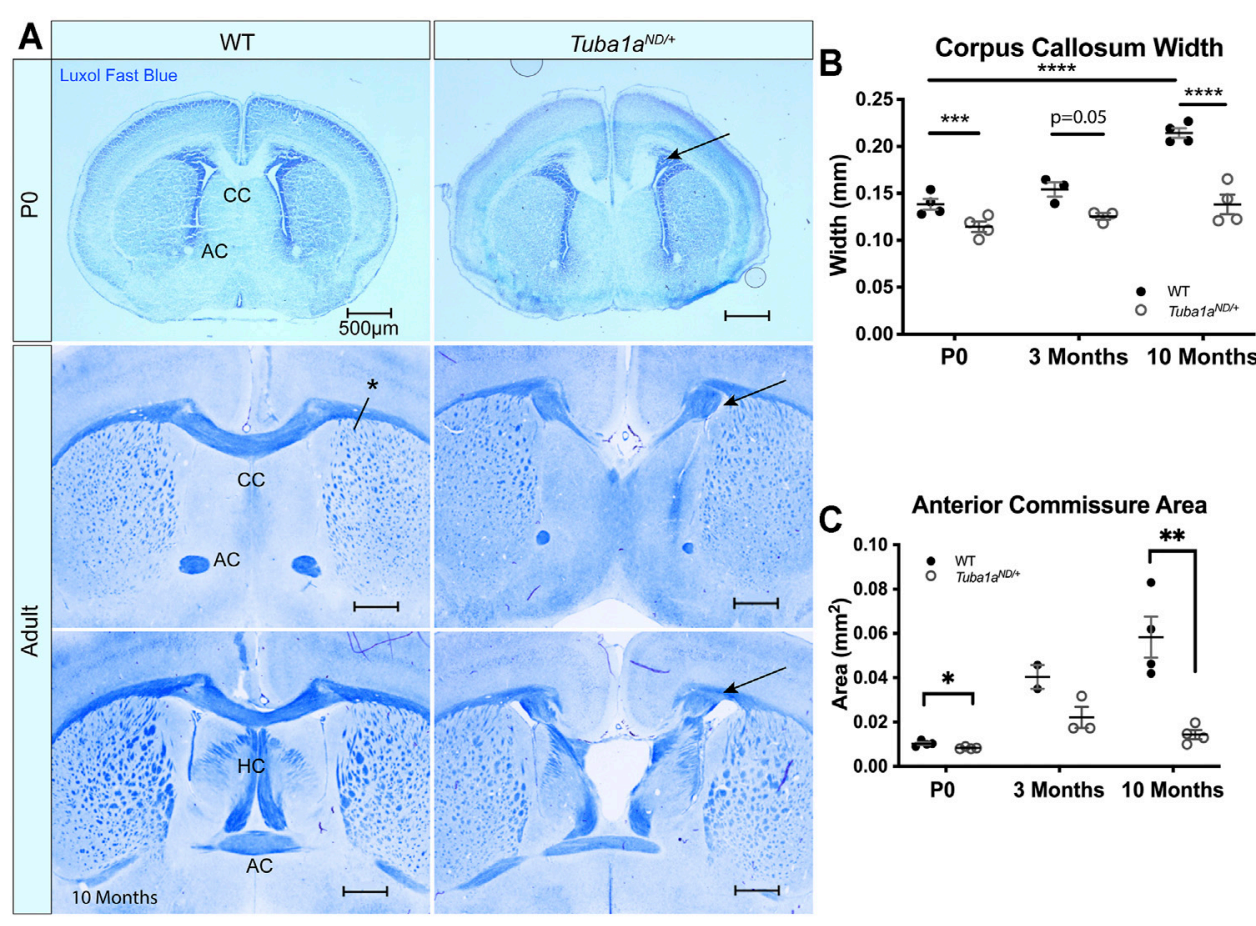

FIGURE 3 | Tuba1a is required for formation of midline commissural structures. (A) Luxol fast blue-stained coronal brain sections from postnatal day 0 (PO; top) and Adult (middle-bottom) wild-type and Tuba1 $\mathrm{N}^{\mathrm{ND} / \mathrm{+}}$ mice. Images portray abnormal midline commissural formation in Tuba $1 \mathrm{a}^{\mathrm{ND} /{ }_{+}}$mouse brains, with labels highlighting the corpus callosum (CC), anterior commissures (AC), and hippocampal commissure (HC). Scale bars are $500 \mu \mathrm{m}$. Arrows indicate Probst bundles. Asterisk in A. shows where measurements for B. were obtained. (B) Scatter plot representing corpus callosum width at PO, 3 months, and 10 months-old. (C) Scatter plot displaying anterior commissure area in P0, 3 months, and 10 months-old wild-type and Tuba1a ${ }^{N D /+}$ brains. Wild-type and Tuba1a ${ }^{N D /+}$ measurements compared by two-way ANOVA. ${ }^{*} p<0.05 ;{ }^{* \star} p<0.01 ;{ }^{* \star *} p<0.001 ;$ and ${ }^{* \star \star *} p<0.0001$.

our results indicate that reduced Tubala is not sufficient to support commissure formation.

\section{Tuba1a is Necessary for Neurite Extension and Cytoskeletal Organization in Growth Cone}

To assess potential mechanisms by which reduced Tubala prevents commissural axons from crossing the midline, we measured neurite lengths, growth rates, and retraction frequency in cultured primary cortical neurons from $\mathrm{P} 0$ wild-type and Tubala $a^{N D /+}$ mice (Figure 5A). Tubal $a^{N D /+}$ neurites were significantly shorter than wild-type neurites ( $32.51 \pm 1.75$ vs. $41.25 \pm 1.71 \mu \mathrm{m}, p=0.0016$ by $t$-test, $n=157$ WT neurons $\mathrm{N}=13$ mice and $77 \mathrm{Tubala} \mathrm{a}^{\mathrm{ND/+}}$ neurons, $\mathrm{N}=9$ mice) at DIV1 (Figure 5B). Tubal $a^{N D /+}$ neurites trended towards slower growth rates $(5.828$ vs. $4.064 \mu \mathrm{m} / \mathrm{h}, p=$ 0.1853 by $t$-test, Figure 5C) and increased frequency of retraction ( 49.25 vs. $53.39 \%, p=0.2247$ by $t$-test, Figure $5 D$ ) in an hour. Measurements of the longest neurite, the putative "axon", at DIV 3 revealed that Tuba1 $a^{N D /+}$ neurites remained significantly shorter than those of wild-type neurons (Figure 5E; $\mathrm{N}=3$ mice, $n=124$ neurons; $p=0.02$ by $t$-test). Together, these data show that developing neurons with reduced Tubala have a diminished capacity for neurite growth compared to wild-type neurons.

To determine if there are differences in the cytoskeleton network that explain shorter Tubal $a^{N D /+}$ neurites, we assessed the abundance of acetylated microtubules and filamentous actin (F-actin) in developing growth cones of wild-type and Tuba1 $a^{N D /+}$ cortical neurons at DIV 3 (Figure 5F). The growth cone is a dynamic developmental structure that uses the coordinated action of the actin and microtubule cytoskeleton to drive neuronal outgrowth in response to internal and external cues (Dent et al., 2011; Craig, 2018). The area of Tubala $a^{N D /+}$ growth cones trended towards reduced area $\left(317.4 \pm 31.3 \mu \mathrm{m}^{2}\right)$ compared to wild-type $\left(380.9 \pm 30.4 \mu \mathrm{m}^{2}\right.$; Figure 5G; $n=49$ growth cones; $p=0.15$, by student's t-test). We examined the amount of acetylated tubulin, a post-translational modification associated with stable microtubules (Figure 5H) and found there to be no difference in the overall fluorescence intensity of acetylated tubulin in Tubal $a^{N D /+}$ neurons compared to wild-type $(n=$ 49 growth cones; $p=0.89$ ). In contrast, we observed a significant increase in F-actin fluorescence intensity within the growth cones of Tuba1 $a^{N D /+}$ neurons compared to wild-type (Figure 5I; $n=49$ growth cones; $p=0.0014)$. Normally, neuronal microtubules splay out in the central, actin-dominated regions of the growth cone, but are bundled towards the peripheral domains of the growth cone (Buck and Zheng, 2002). To assess the degree of growth cone microtubule bundling, we next performed line scans across the widest point of DIV 3 growth cones $\geq 10 \mu \mathrm{m}$ (Figure 5J), an assay that was modeled after similar experiments in Biswas and Kalil (2018) (Biswas and Kalil, 2018). Line scans of acetylated tubulin through the growth cone revealed differences in microtubule organization between wild-type and Tuba1 $a^{N D /+}$ neurons (Figure 5K; $n=39$ growth cones; $p<0.0001$ 


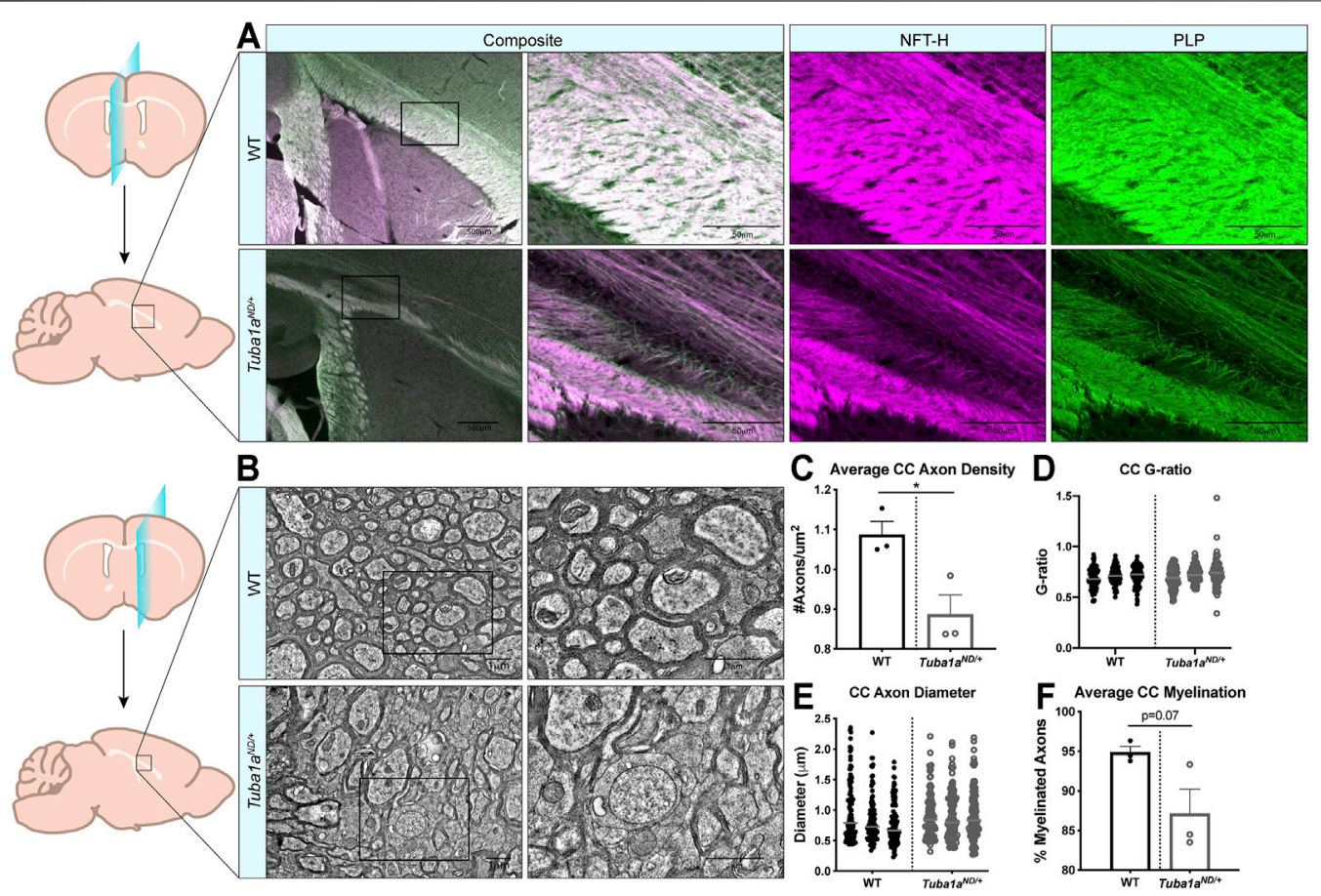

FIGURE 4 | Axon density is reduced by Tuba1 $a^{N D /+}$ in midline corpus callosum. (A) Sagittal brain sections at midline from wild-type (top) and Tuba1a ${ }^{N D /+}$ (bottom) brains stained with neurofilament-heavy (NFT-H; magenta) to label axons, and proteolipid protein (PLP; green) to label myelin. Images were taken at $\times 4$ (left) and $\times 20$ (right) magnification with 500 and $50 \mu \mathrm{m}$ scale bars, respectively. (B) Electron microscopy (EM) images of sagittal wild-type (top) and Tuba1a ND/+ (bottom) sections of corpus callosum $2 \mathrm{~mm}$ adjacent to midline. Enlarged regions on right are denoted by boxes, scale bar is $1 \mu \mathrm{m}$. (C) Scatter plot representing axon density of wildtype and Tuba1 $\mathrm{a}^{\mathrm{ND/+}}$ axons in analyzed EM images. Data points represent average values for each animal $(n=6$ regions containing same total area; $p=0.03)$. (D) Scatter plot of G-ratio measurements for wild-type and Tuba1 $\mathrm{a}^{\mathrm{ND} /+}$ axons. Data points represent individual myelinated axons and are clustered by animal ( $\mathrm{N}=3$ mice, $n=100$ axons; $p=0.34)$. (E) Scatter plot of axon diameters in wild-type and Tuba1 $a^{N D /+}$ corpus callosum by EM. Only those axons captured in cross section were assessed for diameter, as skewed axons provide inaccurate measurements. Data points represent individual axons and are clustered by animal $(n=100 ; p=0.14)$. (F) Scatter plot representing the average percent of myelinated axons per animal in wild-type and Tuba1 $a^{N D /+}$ corpus callosum. Six regions containing the same total area were assessed $(n=6 ; p=0.07)$. Statistical differences between means of wild-type and Tuba1 ${ }^{N D /+}$ datasets were assessed by $t$ test, with ${ }^{\star} p<0.05$.

between genotypes by two-way ANOVA). Specifically, we observed peaks in fluorescence, indicating bundled microtubules, at the edges of the growth cone in wild-type neurons, where acetylated tubulin was more diffuse and lacked obvious organization in Tubal $a^{N D /+}$ growth cones (Figure 5K). The ratio of acetylated microtubules to F-actin in the growth cone was significantly reduced in Tubal $a^{N D /+}$ neurons compared to wild-type, indicating changes to the overall growth cone cytoskeletal environment in Tuba1 $a^{N D /+}$ neurons (Figure 5L; $n=49$ growth cones per genotype; $p=0.0003$ ). Together, these data indicate that neurite growth is particularly sensitive to the amount of Tubala tubulin available, and that reduction in Tubala leads to shorter neurites (a result of subtle changes to growth and retraction rates) with growth cones containing abnormal actin and microtubule architecture.

\section{Tuba1a $^{N D /+}$ Neurites Exhibit Short Term Growth Increase With Global Addition of Netrin-1}

Axon extension to form the corpus callosum is directed by guidance cues, including Netrin-1 (Nishikimi et al., 2013). Netrin-1 can act as an attractive guidance cue for developing axons by promoting directional axonal growth (Boyer and Gupton, 2018), and Netrin-1 deficient mice exhibit defects in commissural axon projection (Serafini et al., 1996) similar to that seen in Tubala $a^{N /+}$ mice (Figure 3). To determine if Tuba1 $a^{N D /+}$ neurons respond to Netrin-1, primary cortical neurons were transfected with a membrane bound tdTomato reporter and cultured for $24 \mathrm{~h}$. At DIV1, time lapse images were taken every $10 \mathrm{~min}$ over the course of an hour, then purified Netrin-1 was added to the media. Time lapse images taken after the addition of Netrin-1 revealed that both wild-type and Tuba1 $a^{N D /+}$ neurons have a slight but significant increased growth rate at $10 \mathrm{~min}$ after the addition of Netrin-1 (Figures 6A,B). No differences were observed when comparing wild-type and Tubal $a^{N D /+}$ neurons directly at the same time points (Figure 6C). These data indicate that both wild-type and Tubala $a^{N D /+}$ neurons exhibit similar short-term responses to global addition of Netrin-1 at DIV1 in culture.

\section{Tuba1a $^{N D}$ Neurons Fail to Localize Critical Developmental Protein Map1b to Growth Cone}

MAPs play a crucial role in regulating neuronal microtubule function to support proper neurodevelopment. Microtubule- 

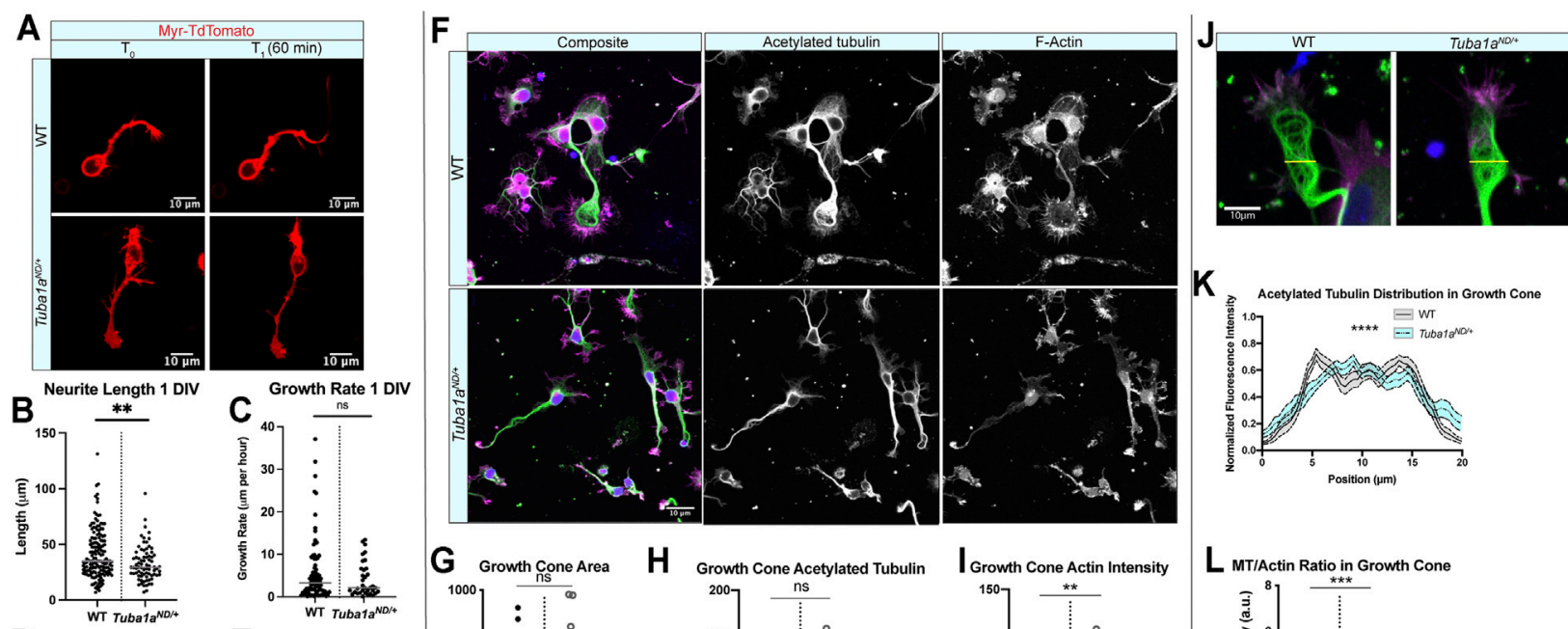

D Retraction Frequency
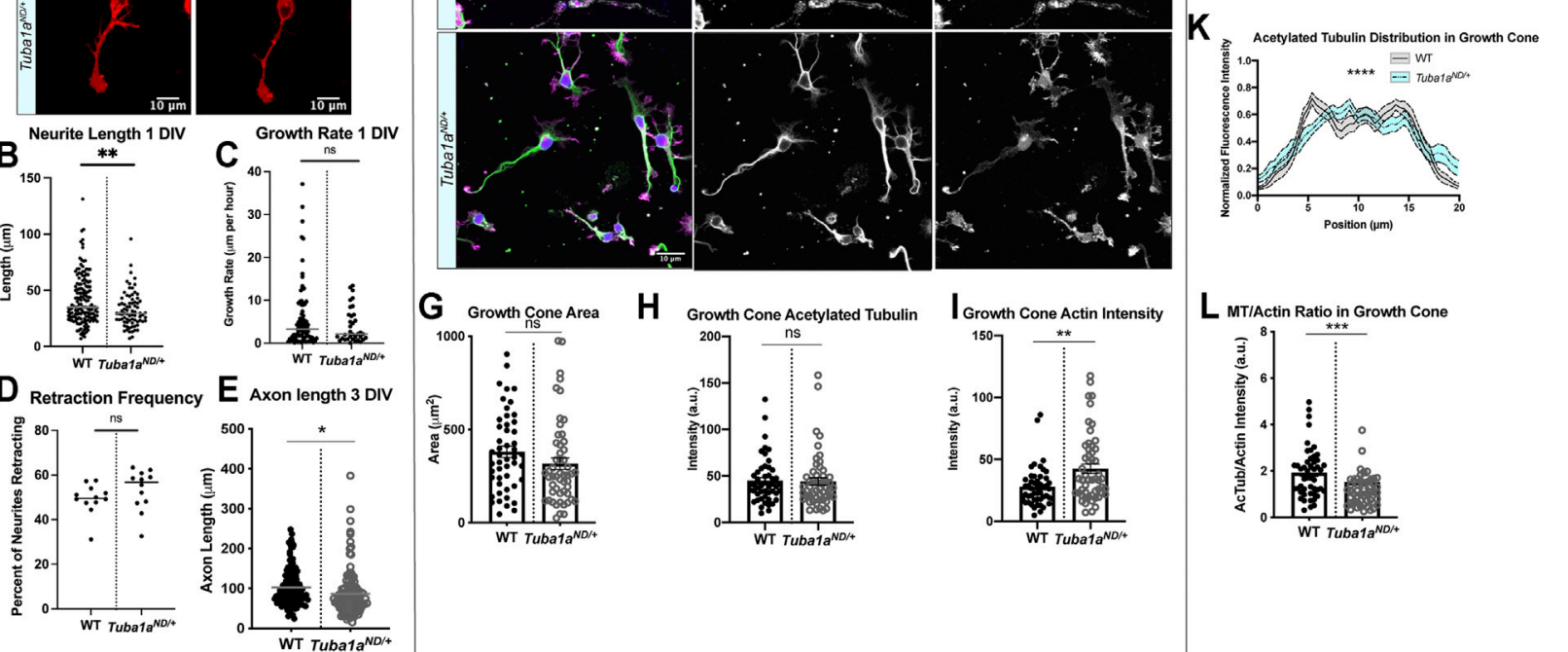

WT Tuba1a ${ }^{N D}$

WT Tuba1a ${ }^{\mathrm{NO} /}$

WT Tuba1a ${ }^{\mathrm{NO} / \mathrm{t}}$

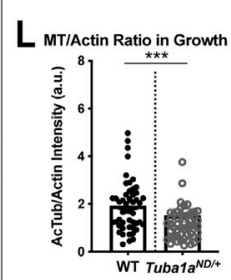

FIGURE 5 | Tuba1 ${ }^{N D}$ impairs neurite outgrowth and alters growth cone cytoskeleton in cortical neurons. (A) Representative time-lapse images of membranelabeled Myr-TdTomato neurons at DIV 1. Myr-TdTomato images were acquired 60 min apart to assess neuronal growth rate in wild-type (WT; top) and Tuba1a ${ }^{\text {ND/+ }}$ (bottom) cortical neurons. (B) Neurite lengths from WT and Tuba1 $\mathrm{a}^{\mathrm{ND} /+}$ mice at DIV 1. Data points represent individual neurons $(32.51 \pm 1.75 \mathrm{vs}$. $41.25 \pm 1.71 \mu \mathrm{m}, p=$ 0.0016 by $t$-test, $n=157$ WT neurons $\mathrm{N}=13$ mice and 77 Tuba1aND/+ neurons, $\mathrm{N}=9$ mice). (C) Scatter plot of neurite growth rate in DIV 1 neurons measured over 60 min using Myr-TdTomato tagged primary cortical neurons from WT and Tuba1 $\mathrm{a}^{\mathrm{ND} /+}$ mice (5.828 vs. $\left.4.064 \mu \mathrm{m} / \mathrm{h}, p=0.1853\right)$. (D) Scatter plot of neurite retraction frequency reveals a trend toward more frequent retractions in Tuba $1 \mathrm{a}^{\mathrm{ND} /+}$ mice $(49.25 \mathrm{vs} .53 .39 \%, p=0.2247)$. (E) Scatter plot of neurite length at DIV 3 in fixed WT and $\mathrm{Tuba}_{1} \mathrm{a}^{\mathrm{ND} / \mathrm{+}}$ primary cortical neurons. For each cell, the longest neurite, designated a putative "axon", was measured from the cell soma to the distal neurite tip using an

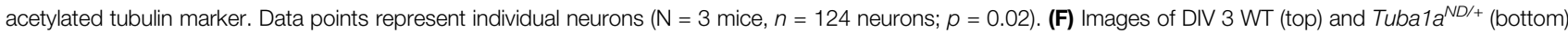
cortical neurons stained with antibodies directed against acetylated tubulin (green) and rhodamine phalloidin (F-actin, magenta). Composite images are shown (left) with single channel grayscale images for acetylated tubulin (middle) and F-actin (right). Fluorescent images were post-processed in ImageJ to enhance brightness and contrast, set to a minimum value of 0 and a maximum value of 70 . Scale bars are $10 \mu \mathrm{m}$. (G) Scatter plot of growth cone area at DIV 3 in WT and Tuba1a ${ }^{\text {ND/+ }}$ cortical neurons. Data points represent individual growth cones $(\mathrm{N}=3$ mice, $n=52$ growth cones; $p=0.26)$. (H) Scatter plot of acetylated tubulin intensity within the growth cone of WT and Tuba1 $\mathrm{a}^{\mathrm{ND} / \mathrm{+}}$ cortical neurons at DIV 3. Acetylated tubulin images were collected using identical microscope imaging settings between samples, fluorescence of quantified images was not adjusted prior to measurement. Acetylated tubulin intensity was normalized to the area of the growth cone. Data points represent individual growth cones ( $n=49$ growth cones; $p=0.89$ ). (I) F-actin intensity within growth cone of WT and Tuba1a ${ }^{N D /+}$ cortical neurons at DIV 3 . F-actin images were collected using identical microscope imaging settings between samples, fluorescence of quantified images was not adjusted prior to measurement. F-actin intensity was normalized to the area of the growth cone. Data points represent individual growth cones ( $\mathrm{N}=3$ mice, $n=49$ growth cones; $p=0.0014$ ). ( $\mathbf{J}$ ) Representative images of WT and Tuba1 $\mathrm{a}^{\mathrm{ND/+}}$ cortical neuron growth cones showing distribution of acetylated tubulin (green) and F-actin (magenta). The yellow line indicates where microtubule fluorescence intensity measurements were taken. (K) Line plot showing the average acetylated tubulin fluorescence intensity across a $20 \mu \mathrm{m}$ line scan through the central domain of the growth cone, taken perpendicular to the extending neurite shaft or putative axon in DIV 3 WT and Tuba1 $a^{N D /+}$ neurons. Differences between WT and Tuba $1 \mathrm{a}^{\mathrm{ND/+}}$ growth cones were assessed by two-way ANOVA which showed a significant interaction between genotype and fluorescence intensity by position ( $n=$ 20 growth cones; $p<0.0001)$. (L). Scatter plot representing the ratio of acetylated tubulin to F-actin intensity within the growth cones of DIV 3 WT and Tuba1a ${ }^{\text {ND/+ }}$ cortical neurons. Acetylated tubulin and F-actin intensity was measured from a single ROI in each growth cone, fluorescent images were acquired using the same microscope settings between samples and were not post-processed to adjust fluorescence. Data points represent individual growth cones ( $\mathrm{N}=3 \mathrm{mice}, n=49$ growth cones; $p=0.0003$ by Mann-Whitney test). For all plots, lines represent mean and error bars report SEM. Differences between WT and Tuba1aND/+ datasets were assessed by $t$ test unless otherwise noted. ${ }^{\star} p<0.05 ;{ }^{\star \star} p<0.01 ;{ }^{\star \star \star} p<0.001 ;{ }^{\star \star \star \star} p<0.0001$

associated protein $1 \mathrm{~b}($ Map1b) regulates microtubule dynamics and facilitates actin/microtubule crosstalk to promote axon extension (Del Río et al., 2004). Mice lacking Map1b display an absence of the corpus callosum (Meixner et al., 2000; Gonzalez-Billault et al., 2001), similar to what is seen in Tubal $a^{N D /+}$ mice. To assess whether Tubala ${ }^{N D}$ might disrupt Maplb function, we performed a series of biochemical and immunocytochemical analyses. Map1b is highly enriched in neurons compared to other cells in the brain, and neuronal expression of Map1b accounts for most of the Map1b in the whole brain (Zhang et al., 2014; Zhang et al., 2016). Western blot analysis of whole brain lysates from wild-type and Tubala $a^{N D /+}$ mouse brains showed no difference in the total amount of Map1b protein (Figure 7A; $p=0.98$ ). There was no significant deficit in the association of Map1b with taxolstabilized microtubules from $T u b a 1 a^{N D /+}$ brain lysates 

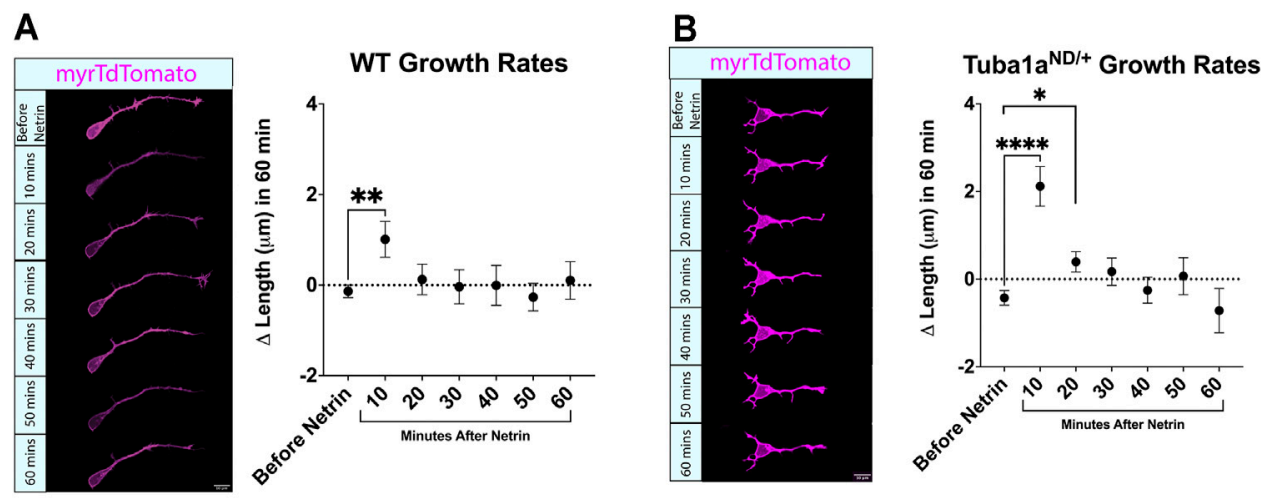

\section{WT vs ND Growth Rate}

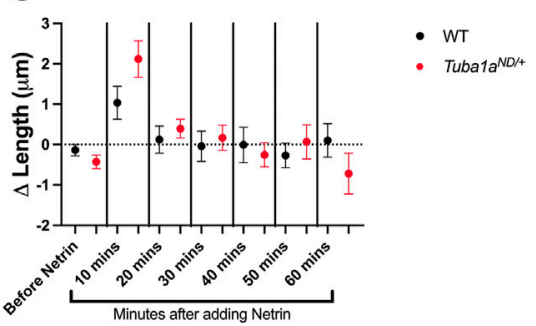

FIGURE 6 | Reduced Tuba1a does not interfere with netrin-1-induced axon growth rates. (A) Primary cortical neuron cell cultures were made from Tuba1 ${ }^{\text {ND/+ }}$ mice and wildtype littermates. Cells were plated on glass-bottom dishes and images were collected every $10 \mathrm{~min}$ for an hour. Purified Netrin-1 was then added to the dish at $500 \mathrm{ng} / \mathrm{ml}$ and images were taken every $10 \mathrm{~min}$ for an additional hour. $\left(n=77\right.$ neurons; $p=0.0057$, unpaired $t$-test) (B). Analysis was repeated for Tuba1 $a^{\mathrm{ND} /+}$ mice $(n=91$ neurons; $p<0.0001$, and $n=83$ neurons; $p=0.0488$, unpaired $t$-test) (C). To determine if there was a difference in stimulated growth between the wildtype and Tuba $1 a^{\mathrm{ND} /+}$ mice the growth values were compared at each time point. No differences were found at any time point.

compared to wild-type; in fact, Tubala $a^{N D /+}$ samples contained slightly more Map1b than wild-type (Figure $7 \mathbf{B} ; p=0.03$ ). In addition, we performed a western blot to quantify and compare the amount of Maplb associated with polymerized tubulin in brains from WT and Tuba $1 a^{N D /+}$ brains without taxol, at $37^{\circ} \mathrm{C}$ to avoid stimulating tubulin polymerization. We found that there was no difference in Map1b in the polymerized tubulin fraction (Figure 7C). These data indicate Tubal ${ }^{N D}$ does not impair the interaction between Maplb and microtubules. In developing wild-type neurons, Map1b localizes strongly to the growth cone to promote axon growth and facilitate microtubule response to guidance cues (Figure 7D) (Black et al., 1994; Mack et al., 2000; Gonzalez-Billault et al., 2001; González-Billault et al., 2002; Tymanskyj et al., 2012). Strikingly, while Tubala $a^{N D /+}$ neurons contained Map1b protein, they exhibited very little Maplb fluorescence in the growth cone compared to wild-type neurons (Figure 7E; $n=31$ growth cones; $p=0.009)$. These data provide evidence that while the abundance of Map1b protein is unchanged by Tuba $1 a^{N D}$, reduced Tubala dramatically impedes the subcellular localization of Map1b to the growth cone. Failure of Tubala $a^{N D /+}$ neurons to localize Map1b to the developing growth cone provides a putative mechanism by which developing axons may fail to correctly organize the growth cone cytoskeleton, leading to the commissural deficits observed in Tubala $a^{N D /+}$ mice.

\section{DISCUSSION}

\section{Functions of TUBA1A During Neurodevelopment}

Using TUBA1A-His6 as a novel imaging tool, we illustrate that the TUBA $1 A^{N D}$ allele is excluded from microtubule filaments and decreases the abundance of TUBA1A in cells (Figures 1, 2). We further demonstrate that mice with diminished Tubala exhibit normal cortical layering but are incapable of forming axon commissures. On a cellular level, decreased Tubala impairs neurite outgrowth (Figures 5-7) and disrupts growth cone localization of Map1b, which acts as a critical regulator of cytoskeletal dynamics during axonal outgrowth (Figure 8). The timing of axonal extension is precisely regulated, and growth cones which fail to reach targets at the correct time can miss crucial developmental signaling events. Interactions between MAPs and microtubules play a major role in adapting microtubule function in response to changing intra- and extra-cellular developmental environments (Meixner et al., 2000; Del Río et al., 2004; Tymanskyj et al., 2012). Therefore, it's possible that neurons with reduced Tubala may be incapable of maintaining appropriate axonal growth rates or mounting appropriate cytoskeletal responses to extracellular guidance cues due to lack of localization of critical cargos such as MAP1B (Figure 8B). Our evidence supports a model in which adequate TUBA1A levels are required for neurite 


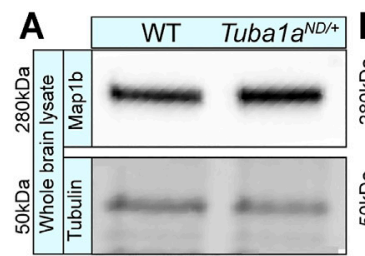

Total Map1b

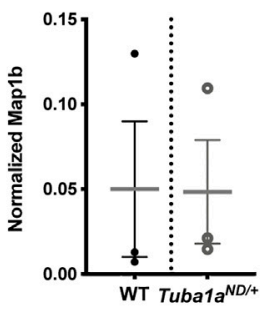

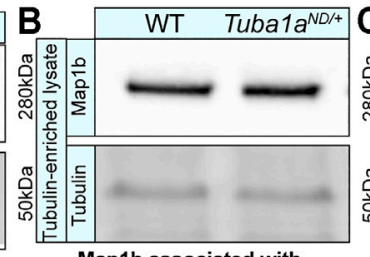

Map1b associated with Tubulin-enriched Fraction

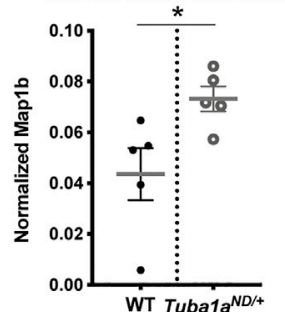

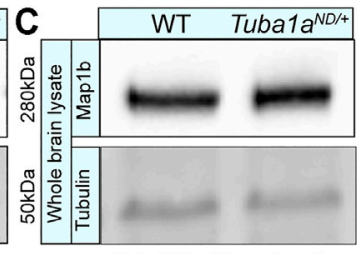

Total Map1b no taxol

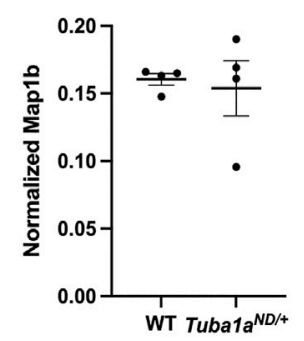

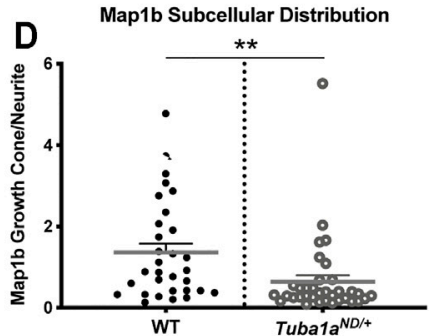
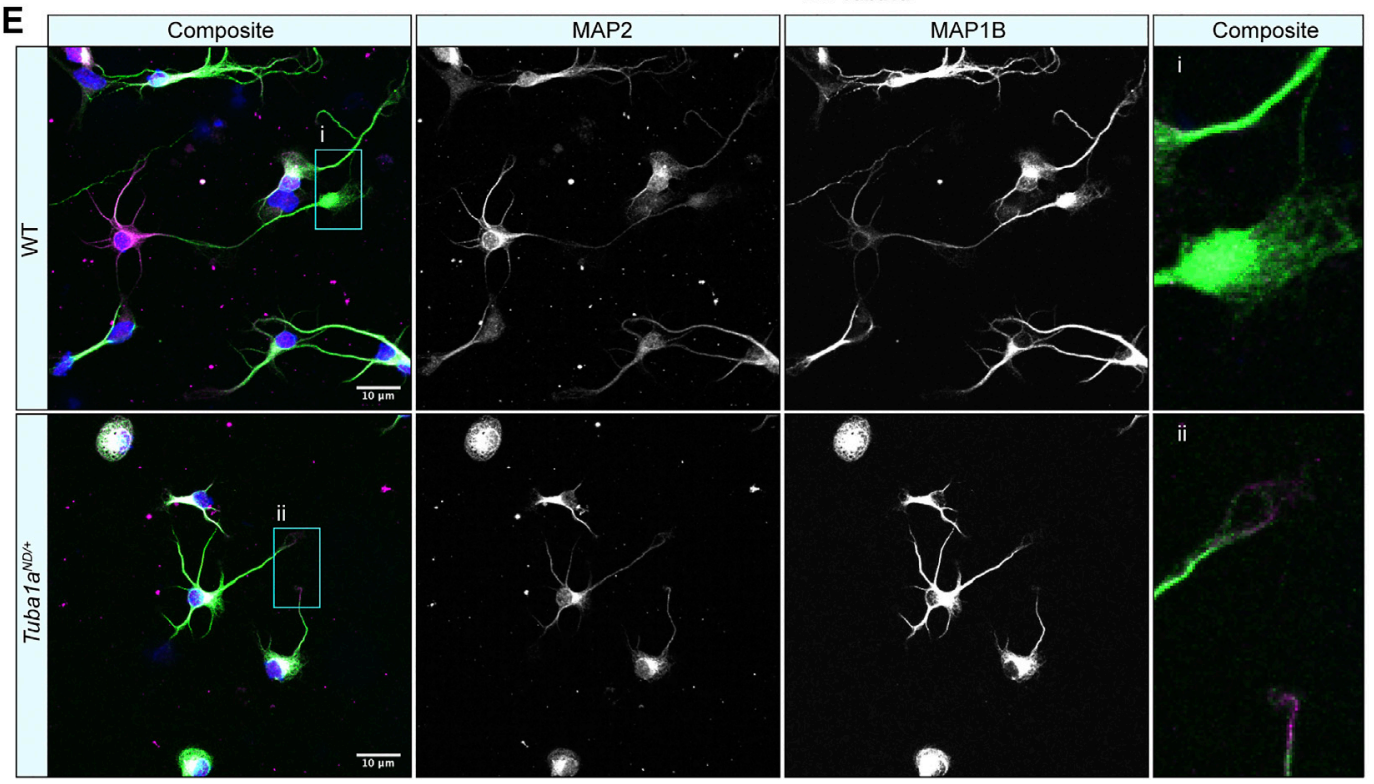

FIGURE 7| Tuba1 $a^{\text {ND }}$ neurons do not correctly localize Map1b to the developing growth cone. (A) Western blot showing total Map1b protein in whole brain lysate from wild-type (WT) and Tuba1 $\mathrm{a}^{\mathrm{ND/+}}$ mice. Scatter plot representing total Map1b protein in brain lysate by western blot, normalized to the total protein on a stain-free blot. $p=0.98$ by $t$-test. (B) Western blots showing Map1b protein associated with a taxol stabilized tubulin-enriched fraction from brain (top panel). Due to the amount of protein that was loaded for Map1b western blots, antibody-stained bands for $a$-tubulin were oversaturated and could not be quantified, thus Map1b was normalized to the $50 \mathrm{kDa}$ band (presumed to be primarily tubulin) on a UV-activated stain-free blot (bottom panel). Scatter plot quantifying Map1b associated with the taxol-stabilized tubulin-enriched brain lysate, normalized to the $50 \mathrm{kDa}$ presumed tubulin band using stain-free western blotting. $p=0.03$ by $t$-test (C). Western blot showing Map1b from WT and Tuba1 $\mathrm{a}^{\mathrm{ND} /+}$ pellet fraction of brain lysates without the addition of taxol. Scatter plot quantifying Map1b associated with tubulin enriched from brain lysates shows no significant difference between the amount of Map1b associated with tubulin when no taxol was added, $p=0.76$ by $t$-test. Map $1 b$ is normalized to the $50 \mathrm{kDa}$ tubulin band using stain-free western blotting. (D) Scatter plot showing the subcellular distribution of Map1b protein in WT and Tuba1a ND/+ cortical neurons at DIV 3. Data are represented as Map1b fluorescent signal in growth cone region divided by a region proximal to the cell body of the same area. Fluorescent images were acquired using identical microscope imaging settings between samples and were not post-processed to adjust fluorescence prior to quantification. $p=0.009$. (E) Representative images showing altered subcellular distribution of Map $1 \mathrm{~b}$ in Tuba1 $\mathrm{a}^{\mathrm{ND/+}}$ (bottom) cortical neurons compared to WT (top) at DIV 3. Composite and individual channel grayscale images of MAP2 and Map1b immunocytochemistry are shown, $i$ and ii indicate enlarged regions shown in insets. Scale bars are $10 \mathrm{~m}$. Differences between groups were evaluated by $t$ test. ${ }^{*} p<0.05 ;{ }^{\star \star} p<0.01$.

extension and commissure formation, which is achieved via a dynamic cytoskeleton that can readily respond to external cues through the action of critical factors localized to the growth cone. Without sufficient Tubala, axons cannot grow at the appropriate rate or correctly localize important $\mathrm{MAP}(\mathrm{s})$, rendering Tubala deficient axons incapable of crossing the midline to reach distant targets.

\section{Studying a-Tubulin Isotypes}

TUBA1A has long been associated with neurodevelopment due to its spatial and temporal expression as well as its causal role in tubulinopathies (Miller et al., 1987; Gloster et al., 1994; Bamji and Miller, 1996; Gloster et al., 1999; Poirier et al., 2007; FalletBianco et al., 2008; Kumar et al., 2010; Lecourtois et al., 2010; Oegema et al., 2015; Aiken et al., 2017), but cellular visualization of a 


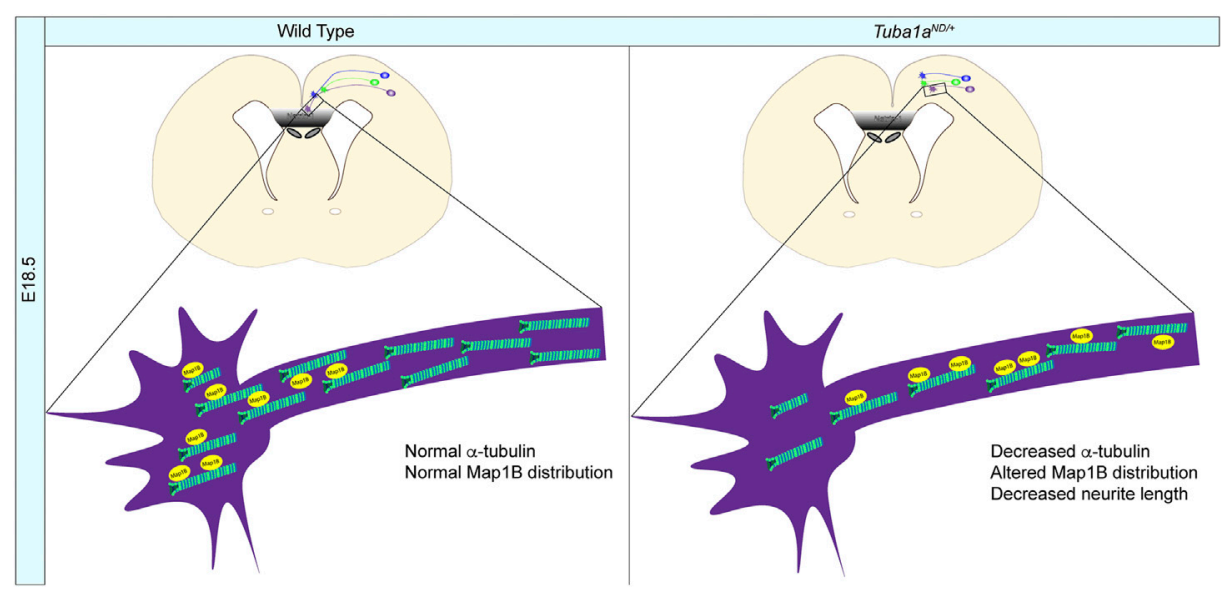

FIGURE 8| Mechanisms of Tuba1a-induced axonal pathfinding deficits. (A) Schematic illustrating how reduced Tuba1a may impair ability of Tuba1a ND/+ axons to reach key signaling points at the correct developmental time to support proper brain formation. Wild-type axons (WT; left) reaching the midline crossing point of corpus callosum by embryonic day (E) 18.5 are compared to the potentially stunted axonal growth in Tuba1a ${ }^{N D /+}$ brains (right). The reduced density of microtubules impair localization of Map1b and potentially other MAPs to contribute to slower growth of developing Tuba1a ${ }^{N D /+}$ commissural neurons.

single $\alpha$-tubulin isotype or mutant has remained elusive due to the limited availability of isotype-specific tools. Studying individual $\alpha$ tubulin isotypes in neurons has been historically arduous as the high degree of amino acid sequence similarity between $\alpha$-tubulin isotypes (TUBA1A and TUBA1B proteins are $99.5 \%$ identical) leads to antibody promiscuity and has made genetically targeting a single a-tubulin gene challenging. The abundance of clinically identified mutations to $T U B A 1 A$ provide strong evidence that $T U B A 1 A$ is a major player in both tubulinopathy and typical neurodevelopment; however, the lack of available tools to study TUBA1A in cells has prevented researchers from understanding precisely how TUBA1A contributes to neurodevelopment. As such, previous studies of tubulinopathy mutations have relied heavily on mRNA analysis and indirect methods of evaluating TUBA1A protein function. Here we harness a previously-identified internal loop within TUBA1A that tolerates addition of small epitope tags without impacting TUBA1A incorporation or dynamics (Schatz et al., 1987; Heilemann et al., 2008; Hotta et al., 2016) to generate an important tool for the study of tubulinopathies and neuronal $\alpha$ tubulin. Advantages of this tool are many. In Cos-7 cells and neurons, wild-type TUBA1A-His6 was able to incorporate into microtubule polymers, unlike TUBA1A containing a GFP fusion that prohibited incorporation into neuronal microtubules (Figure 2). Further, internal tagging does not interfere with C-terminal post translational modifications, which are abundant in neurons and contribute to function. The His6 tag can also be eluted natively so that the same tubulin isotype mutants can be purified for in vitro studies to complement localization and functional studies completed in cells. Additionally, the addition of the internal His6 tag did not remove or replace the Lysine 40 acetylation site. In Cos-7 cells and neurons, wild-type TUBA1A-His6 was able to incorporate into microtubule polymers, unlike TUBA1A containing a GFP fusion that prohibited incorporation into neuronal microtubules (Figure 2). Importantly, this epitope-tagged TUBA1A can be used to gain insight into how mutant tubulin behaves in cellular microtubule networks (Figures 1, 2). For example, we reveal that $T U B A 1 A^{T 349 E}$, a substitution analogous to a previously described mutation that prevents tubulin polymerization in yeast (Johnson et al., 2011), fails to polymerize into neuronal microtubules but does not interfere with endogenous microtubule networks, as revealed by intact acetylated microtubules (Figure $2 \mathrm{C}$ ). In contrast, $T U B A 1 A^{E 255 A}$, a lethal $\alpha$-tubulin stabilizing mutation discovered in yeast which prevents tubulin depolymerization (Anders and Botstein, 2001; Roostalu et al., 2020), is abundantly incorporated into microtubule polymers and increases acetylated tubulin staining compared to wild type (Figure 2C). This mutation leads to thickened microtubule-rich protrusions (Figure 2C). Overall, this tool enables researchers to gain valuable insight into the cellular function of specific a-tubulin isotypes and determine how mutations impact tubulin function, microtubule networks, and cellular morphology.

When applied to TUBA1A ${ }^{N 102 D}$, the epitope-tagged TUBA1A visualization revealed that the N102D mutation leads to polymerization-incompetent tubulin similar to the T349E substitution. Ectopic expression of TUBA1A-His6 protein in cells revealed that TUBA1A ${ }^{\mathrm{ND}}$ protein is excluded from microtubule filaments (Figures 1B, 2C) and is approximately half as abundant as wild-type (Figure 1D), despite similar amounts of mRNA expression in transfected cells (Figure 1E). Based on this evidence, TUBA1 $A^{N D}$ substitution lowers the total amount of usable $\alpha$-tubulin in the cell (Figure 1D). Newly synthesized $\alpha$ and $\beta$-tubulin proteins enter a complex tubulin folding pathway, where they interact with cytosolic chaperonins and tubulin-binding cofactors to become folded and assembled into tubulin heterodimers (Lewis et al., 1997). We found TUBA1 ${ }^{N D}$ protein to be diminished compared to wild-type, and the introduction of the ND substitution into the primary yeast $\alpha$-tubulin, $T u b 1,\left(T u b 1^{N D}\right)$ is synthetic lethal when combined with tubulin folding pathway mutants. These data reveal an increased reliance on tubulin assembly cofactors when the ND mutation is present, which may be retained in mammalian systems (Gartz Hanson et al., 2016). 


\section{Tuba1a Influences the Microtubule Cytoskeleton in Cells}

The microtubule cytoskeleton supports a wide range of different cellular functions in different cell types, ranging from facilitating chromosome segregation during mitosis to forming dynamic and motile structures such as the neuronal growth cone. Understanding how different cells use the same basic building blocks to create vastly different microtubule-based structures is a major question in microtubule biology. Many different mechanisms have been identified by which cells regulate microtubule network properties and overall function. In this study, we provide the first evidence that TUBA1A is essential for regulating neuronal microtubule function to support commissure formation (Figures 3, 4). Tubal $a^{N D}$ neurons exhibit deficits in neurite extension, [Figures 5, 6; (Buscaglia, 2020)], and do not support growth cone localization of at least one critical developmental MAP necessary for commissural axon pathfinding, Map1b (Figure 6). Collectively, these data support the conclusion that reduced Tubala is sufficient for certain microtubule-dependent neurodevelopmental stages, such as cortical neuron migration (Gartz Hanson et al., 2016), but does not allow for sufficient microtubule function to properly localize proteins to the growth cone or to grow at the rate necessary to form commissures. Thus, axons extending to reach distant targets may be exquisitely sensitive to $\alpha$-tubulin levels.

We previously showed that while there was an overall reduction in a-tubulin protein in brains of heterozygous Tubala $a^{N D /+}$ mice compared to wild type, there was no significant difference in the overall ratio of post-translational modifications, normalized to total $\alpha$-tubulin (Buscaglia, 2020). Here, we show that the Tubala ${ }^{N D}$ mutation changes the distribution of acetylated microtubules subcellularly in the growth cone (Figure 5), likely reflecting altered microtubule organization (Figure 5). Acetylation is a microtubule PTM that is associated with stable microtubule populations and as such is sparse in dynamic structures like growth cones (Schulze et al., 1987; Robson and Burgoyne, 1989; Mansfield and GordonWeeks, 1991; Palazzo et al., 2003; Baas et al., 2016; Eshun-Wilson et al., 2019). However, microtubule acetylation can be induced in growth cones following contact with extracellular matrix proteins and promotes cortical neuron migration in vivo and suppresses axon branching in vitro, demonstrating a clear role for this PTM in development (Dan et al., 2018; Bergstrom et al., 2007; Creppe et al., 2009). Tubulin PTMs, like acetylation, impact MAPbinding affinity and function, providing a clear mechanism by which changing the PTM landscape of microtubules could alter neuronal microtubule function (Mansfield and Gordon-Weeks, 1991; Bonnet et al., 2001; Reed et al., 2006; Janke and Kneussel, 2010; Lacroix et al., 2010; Sirajuddin et al., 2014; Balabanian et al., 2017). Thus, any changes to the organization or distribution of acetylated microtubules in the growth cone could impact the ability of developing neurons to appropriately navigate their environment and establish correct synaptic targets.

Tuba1 $a^{N D /+}$ growth cones showed a significant increase in F-actin fluorescence compared to wild-type, causing an overall shift in the growth cone microtubule-actin balance (Figure 5). This may be due to a shift in distribution of F-actin rather than an increase in levels of F-actin. It is well established that interplay between the actin and microtubule cytoskeleton drives growth cone movements in developing neurons (Dent and Kalil, 2001; Dent et al., 2011; Piper et al., 2015; Craig, 2018). Growth cone microtubule polymerization induces F-actin assembly, and coordination of actin and microtubules is regulated by interactions with MAPs to drive appropriate growth cone response (Rochlin et al., 1999; Szikora et al., 2017; Biswas and Kalil, 2018; Slater et al., 2019). As actin and microtubules are tightly regulated within the growth cone, it is reasonable to assume that mutations which disrupt microtubule function, like $T u b a 1 a^{N D}$, likely also impact the actin cytoskeleton of developing neurons. In Tuba1 $a^{N D /+}$ neurons, the actin cytoskeleton may occupy increased growth cone territory as the result of microtubule deficiencies, but additional testing of actin-response in developing Tubal $a^{N D /+}$ neurons is needed to assess whether the increase in growth cone actin has any functional consequences.

We show that Tuba $1 a^{N D /+}$ neurons do not effectively localize at least one developmental MAP, Map1b, to the growth cone (Figure 7). We previously demonstrated that axonal transport is impaired in developing Tubala $a^{N D /+}$ neurons (Buscaglia, 2020), thus reduced Tubala could lead to altered localization of developmental MAPs due to disrupted trafficking. Our data do not support the conclusion that Maplb expression or binding to microtubules is reduced. Instead, we favor a model in which trafficking is disrupted so that Map1b is not appropriately localized. Intracellular transport is a crucial function of neuronal microtubules throughout life, and microtubule-based transport is essential during neurodevelopment for distributing cellular components into burgeoning neurites (Hoogenraad and Bradke, 2009; Feltrin et al., 2012; Liu et al., 2012; Baraban et al., 2013; Dent and Baas, 2014; Leung et al., 2018; Miller and Suter, 2018). Correct localization of developmental MAPs, mRNAs and organelles are necessary for cytoskeletal response to extracellular guidance cues (Welshhans and Bassell, 2011; Feltrin et al., 2012; Qu et al., 2013; Pilaz et al., 2016; Leung et al., 2018). In particular, Map1b is required for commissure formation (Meixner et al., 2000) and homozygous Maplb knockout animals have similar phenotypes to Tubala $a^{N D /+}$ mice [Figure 3 and (Meixner et al., 2000)]. Map1b is necessary for neuronal response to the guidance cue, Netrin1, a key player in commissural formation (Serafini et al., 1996; Barallobre et al., 2000; Finger et al., 2002; Lindwall et al., 2007; Fothergill et al., 2014; Arbeille and Bashaw, 2018). While our data show that Tubal $a^{N D /+}$ neurites grow faster in response to global application of Netrin-1 similar to wild type neurons in the time frame we measured, we cannot rule out impairment of turning in response to a localized source of Netrin1 or response to other guidance cues. With the exception of tubulin and actin, Map1b is the most abundant cytoskeletal protein in the growth cone and localizes asymmetrically to the growth cone (Mack et al., 2000; Igarashi, 2014). Our data suggests that if Map1b is not localized to the growth cone, it cannot perform its function in commissure formation. Map1b acts downstream of several important developmental signaling pathways to regulate function of both actin and microtubules within the growth cone, and dysregulation of this or other MAPs 
could therefore impact multiple cytoskeletal components (Mack et al., 2000; Noiges et al., 2002; Tymanskyj et al., 2012).

\section{Reduced Tuba1a Function does not Adequately Support Neurite Growth for Development}

The timing of axon growth is crucial for the growth cone to receive and respond to the appropriate guidance cues (Rossi et al., 2017; Krol and Feng, 2018). We showed that neuronal microtubules with reduced Tubala do not support axonal growth to the same degree as wild-type microtubules, causing shorter neurite length (Figure 5). If neurons lacking appropriate levels of TUBA1A do not reach the correct location at the correct time, it is possible that neurons will fail to receive key developmental signals (Figure 8). How does reducing Tubala function impair axon extension? One possibility is that there is not enough a-tubulin in the neurite or axon to assemble and extend microtubules. Another, non-exclusive possibility is that the microtubule tracks formed in Tubala $a^{N D}$ axons are not sufficient to support trafficking of microtubule stabilizing factors. Microtubule-based transport is essential during neurodevelopment (Hoogenraad and Bradke, 2009; Feltrin et al., 2012; Liu et al., 2012; Baraban et al., 2013; Dent and Baas, 2014; Leung et al., 2018; Miller and Suter, 2018), and we previously showed that intracellular transport is impaired in developing Tuba1 ${ }^{N D /+}$ neurons (Buscaglia, 2020). Map1b is not appropriately localized to the growth cone (Figures 7, 8), suggesting that diminished Tubala function in neurons impacts the subcellular localization of Map1b, and potentially other MAPs, to the growth cone through impairments to intracellular trafficking (Figure 8).

Human neurodevelopmental disorders that impact microtubule function, such as tubulinopathies, demonstrate that microtubules are critical for neurodevelopment. Tubulinopathy patients exhibit severe, sometimes lethal, brain malformations that frequently impact multiple neurodevelopmental processes, including neuronal survival, migration, and axon extension (Fallet-Bianco et al., 2008; Tian et al., 2008; Keays et al., 2010; Kumar et al., 2010; Tian et al., 2010; Bamba et al., 2016; Belvindrah, 2017; Aiken et al., 2019a; Aiken et al., 2019b). The range of phenotypes exhibited by tubulinopathy patients have made it challenging for scientists to pinpoint specific aspects of neuronal function that are reliant on TUBA1A tubulin. We used Tubala $a^{N D}$ as a tool to interrogate the requirement for Tubala in discrete aspects of neurodevelopment. Importantly, commissural abnormalities, such as agenesis of the corpus callosum, are commonly reported features in tubulinopathy patients (Poirier et al., 2007; Fallet-Bianco et al., 2008; Cushion et al., 2013; Oegema et al., 2015; Aiken et al., 2017). Cortical malformations and neuronal migration errors are also common features of TUBA1A tubulinopathies; however, it has thus far been unclear whether commissural deficits occur as a primary or secondary consequence of TUBA1A dysfunction. In this study, we provide evidence that axons deficient in Tubala fail to properly navigate to meet contralateral binding partners. These data demonstrate that
TUBA1A is required for forebrain commissural formation, independent of its role in neuronal survival or migration.

\section{DATA AVAILABILITY STATEMENT}

The raw data supporting the conclusion of this article will be made available by the authors, without undue reservation.

\section{ETHICS STATEMENT}

The animal study was reviewed and approved by University of Colorado Anschutz Medical Campus IACUC committee.

\section{AUTHOR CONTRIBUTIONS}

GB and EB designed experiments. GB and $\mathrm{KN}$ performed the majority of the experiments and each wrote first drafts of portions of the manuscript. JA generated the Tuba1a-His6 constructs and together with $\mathrm{KH}$ imaged neurons that expressed wild-type or mutant Tuba1a-His6. All authors read, provided feedback and edits to the manuscript. EB directed, and obtained funding for research and edited versions of the manuscript.

\section{ACKNOWLEDGMENTS}

We would like to thank Dr. Jeffrey K. Moore for discussions and valuable advice. We thank Matthew Kennedy and Mark del Acqua (University of Colorado School of Medicine) for providing $\mathrm{P} 0-2$ rat cortex. We would like to thank funding sources without which our work would not be possible: NIH NIDCR R01DE025311 to EAB, NSF-IOS 1945916 to EAB, University of Colorado Movement Disorder Center to EAB, T32 NS099042 to GB, NSF Graduate Research Fellowship 1553798 to JA, NINDS F32NS117672 to JA, T32GM141742-01 to KN. T32 GM136444 to KH. Emily Bates would like to thank the teachers at her children's schools and Bright Horizons Fitzimons daycare during the Covid-19 pandemic without whom, none of her work could be performed.

\section{SUPPLEMENTARY MATERIAL}

The Supplementary Material for this article can be found online at: https://www.frontiersin.org/articles/10.3389/fcell.2021.789438/ full\#supplementary-material

Supplementary Figure S1 | dSTORM imaging of TUBA1A-His6 signal reveals single microtubules. (A) Wide-field (top panels) and dSTORM super-resolution (lower panels and insets) microscopy of DIV7 neurons transfected with TUBA1AHis6 plasmid DNA and immunolabeled with $\alpha$-His6 antibody. Yellow boxes reveal the location of the magnified insets below. (B) dSTORM super-resolution microscopy of DIV4 astrocytes immunolabeled with total $\alpha$-tubulin antibody (left panels) or transfected with TUBA1A-His6 plasmid DNA and immunolabeled with $\alpha$ His 6 antibody. Yellow boxes reveal the location of the magnified insets below. 


\section{REFERENCES}

Aiken, J., Buscaglia, G., Bates, E. A., and Moore, J. K. (2017). The $\alpha$-Tubulin Gene TUBA1A in Brain Development: A Key Ingredient in the Neuronal Isotype Blend. J. Dev. Biol. 5 (3). doi:10.3390/jdb5030008

Aiken, J. (2019). Tubulin Mutations in Brain Development Disorders: Why Haploinsufficiency Does Not Explain TUBA1A Tubulinopathies. Hoboken): Cytoskeleton.

Aiken, J., Moore, J. K., and Bates, E. A. (2019). TUBA1A Mutations Identified in Lissencephaly Patients Dominantly Disrupt Neuronal Migration and Impair Dynein Activity. Hum. Mol. Genet. 28 (8), 1227-1243. doi:10.1093/hmg/ ddy 416

Anders, K. R., and Botstein, D. (2001). Dominant-Lethal $\alpha$-Tubulin Mutants Defective in Microtubule Depolymerization in Yeast. $M B o C 12$ (12), 3973-3986. doi:10.1091/mbc.12.12.3973

Arbeille, E., and Bashaw, G. J. (2018). Brain Tumor Promotes Axon Growth across the Midline through Interactions with the Microtubule Stabilizing Protein Apc2. Plos Genet. 14 (4), e1007314. doi:10.1371/journal.pgen.1007314

Baas, P. W., Rao, A. N., Matamoros, A. J., and Leo, L. (2016). Stability Properties of Neuronal Microtubules. Cytoskeleton 73 (9), 442-460. doi:10.1002/cm.21286

Balabanian, L., Berger, C. L., and Hendricks, A. G. (2017). Acetylated Microtubules Are Preferentially Bundled Leading to Enhanced Kinesin-1 Motility. Biophysical J. 113 (7), 1551-1560. doi:10.1016/j.bpj.2017.08.009

Bamba, Y., Shofuda, T., Kato, M., Pooh, R. K., Tateishi, Y., Takanashi, J.-i., et al. (2016). In Vitro characterization of Neurite Extension Using Induced Pluripotent Stem Cells Derived from Lissencephaly Patients with TUBA1A Missense Mutations. Mol. Brain 9 (1), 70. doi:10.1186/s13041-016-0246-y

Bamji, S. X., and Miller, F. D. (1996). Comparison of the Expression of a T?1:nlacZ Transgene and T?1 ?-tubulin mRNA in the Mature central Nervous System. J. Comp. Neurol. 374 (1), 52-69. doi:10.1002/(sici)1096-9861(19961007)374: $1<52$ :aid-cne4>3.0.co; 2 -m

Baraban, M., Anselme, I., Schneider-Maunoury, S., and Giudicelli, F. (2013). Zebrafish Embryonic Neurons Transport Messenger RNA to Axons and Growth Cones In Vivo. J. Neurosci. 33 (40), 15726-15734. doi:10.1523/ jneurosci.1510-13.2013

Barallobre, M. J., Del Rio, J. A., Alcantara, S., Borrell, V., Aguado, F., Ruiz, M., et al. (2000). Aberrant Development of Hippocampal Circuits and Altered Neural Activity in Netrin 1-deficient Mice. Development 127 (22), 4797-4810. doi:10.1242/dev.127.22.4797

Belvindrah, R. (2017). Mutation of the Alpha-Tubulin Tubala Leads to Straighter Microtubules and Perturbs Neuronal Migration. J. Cel Biol.

Bergstrom, R. A., Sinjoanu, R. C., and Ferreira, A. (2007). Agrin Induced Morphological and Structural Changes in Growth Cones of Cultured Hippocampal Neurons. Neuroscience 149 (3), 527-536. doi:10.1016/ j.neuroscience.2007.08.017

Biswas, S., and Kalil, K. (2018). The Microtubule-Associated Protein Tau Mediates the Organization of Microtubules and Their Dynamic Exploration of ActinRich Lamellipodia and Filopodia of Cortical Growth Cones. J. Neurosci. 38 (2), 291-307. doi:10.1523/jneurosci.2281-17.2017

Bittermann, E., Abdelhamed, Z., Liegel, R. P., Menke, C., Timms, A., Beier, D. R., et al. (2019). Differential Requirements of Tubulin Genes in Mammalian Forebrain Development. Plos Genet. 15 (8), e1008243. doi:10.1371/ journal.pgen.1008243

Black, M., Slaughter, T., and Fischer, I. (1994). Microtubule-associated Protein 1b (MAP1b) Is Concentrated in the Distal Region of Growing Axons. J. Neurosci. 14 (2), 857-870. doi:10.1523/jneurosci.14-02-00857.1994

Bonnet, C., Boucher, D., Lazereg, S., Pedrotti, B., Islam, K., Denoulet, P., et al. (2001). Differential Binding Regulation of Microtubule-Associated Proteins MAP1A, MAP1B, and MAP2 by Tubulin Polyglutamylation. J. Biol. Chem. 276 (16), 12839-12848. doi:10.1074/jbc.m011380200

Boyer, N. P., and Gupton, S. L. (2018). Revisiting Netrin-1: One Who Guides (Axons). Front. Cel. Neurosci. 12, 221. doi:10.3389/fncel.2018.00221

Buck, K. B., and Zheng, J. Q. (2002). Growth Cone Turning Induced by Direct Local Modification of Microtubule Dynamics. J. Neurosci. 22 (21), 9358-9367. doi:10.1523/jneurosci.22-21-09358.2002

Buscaglia, G. (2020). Reduced TUBA1A Tubulin Causes Defects in Trafficking and Impaired Adult Motor Behavior. Washington, D.C.: eNeuro.
Craig, E. M. (2018). Model for Coordination of Microtubule and Actin Dynamics in Growth Cone Turning. Front. Cel. Neurosci. 12, 394. doi:10.3389/ fncel.2018.00394

Creppe, C., Malinouskaya, L., Volvert, M.-L., Gillard, M., Close, P., Malaise, O., et al. (2009). Elongator Controls the Migration and Differentiation of Cortical Neurons through Acetylation of $\alpha$-Tubulin. Cell 136 (3), 551-564. doi:10.1016/ j.cell.2008.11.043

Cushion, T. D., Dobyns, W. B., Mullins, J. G., Stoodley, N., Chung, S. K., Fry, A. E., et al. (2013). Overlapping Cortical Malformations and Mutations in TUBB2B and TUBA1A. Brain 136 (Pt 2), 536-548. doi:10.1093/brain/aws338

Dan, W., Gao, N., Li, L., Zhu, J. X., Diao, L., Huang, J., et al. (2018). a-Tubulin Acetylation Restricts Axon Overbranching by Dampening Microtubule PlusEnd Dynamics in Neurons. Cereb. Cortex 28 (9), 3332-3346. doi:10.1093/ cercor/bhx225

Del Río, J. A., González-Billault, C., Ureña, J. M., Jiménez, E. M., Barallobre, M. J. Pascual, M., et al. (2004). MAP1B Is Required for Netrin 1 Signaling in Neuronal Migration and Axonal Guidance. Curr. Biol. 14 (10), 840-850. doi:10.1016/j.cub.2004.04.046

Dent, E. W., Gupton, S. L., and Gertler, F. B. (2011). The Growth Cone Cytoskeleton in Axon Outgrowth and Guidance. Cold Spring Harb Perspect. Biol. 3 (3). doi:10.1101/cshperspect.a001800

Dent, E. W., and Baas, P. W. (2014). Microtubules in Neurons as Information Carriers. J. Neurochem. 129 (2), 235-239. doi:10.1111/jnc.12621

Dent, E. W., and Kalil, K. (2001). Axon Branching Requires Interactions between Dynamic Microtubules and Actin Filaments. J. Neurosci. 21 (24), 9757-9769. doi:10.1523/jneurosci.21-24-09757.2001

Eshun-Wilson, L., Zhang, R., Portran, D., Nachury, M. V., Toso, D. B., Löhr, T., et al. (2019). Effects of a-tubulin Acetylation on Microtubule Structure and Stability. Proc. Natl. Acad. Sci. USA 116 (21), 10366-10371. doi:10.1073/ pnas. 1900441116

Fallet-Bianco, C., Loeuillet, L., Poirier, K., Loget, P., Chapon, F., Pasquier, L., et al. (2008). Neuropathological Phenotype of a Distinct Form of Lissencephaly Associated with Mutations in TUBA1A. Brain 131 (Pt 9), 2304-2320. doi:10.1093/brain/awn155

Feltrin, D., Fusco, L., Witte, H., Moretti, F., Martin, K., Letzelter, M., et al. (2012). Growth Cone MKK7 mRNA Targeting Regulates MAP1b-dependent Microtubule Bundling to Control Neurite Elongation. Plos Biol. 10 (12), e1001439. doi:10.1371/journal.pbio.1001439

Findeisen, P., Mühlhausen, S., Dempewolf, S., Hertzog, J., Zietlow, A., Carlomagno, T., et al. (2014). Six Subgroups and Extensive Recent Duplications Characterize the Evolution of the Eukaryotic Tubulin Protein Family. Genome Biol. Evol. 6 (9), 2274-2288. doi:10.1093/gbe/evu187

Finger, J. H., Bronson, R. T., Harris, B., Johnson, K., Przyborski, S. A., and Ackerman, S. L. (2002). The Netrin 1 ReceptorsUnc5h3andDccAre Necessary at Multiple Choice Points for the Guidance of Corticospinal Tract Axons. J. Neurosci. 22 (23), 10346-10356. doi:10.1523/jneurosci.22-2310346.2002

Fothergill, T., Donahoo, A.-L. S., Douglass, A., Zalucki, O., Yuan, J., Shu, T., et al. (2014). Netrin-DCC Signaling Regulates Corpus Callosum Formation through Attraction of Pioneering Axons and by Modulating Slit2-Mediated Repulsion. Cereb. Cortex 24 (5), 1138-1151. doi:10.1093/cercor/bhs395

Gadadhar, S., Bodakuntla, S., Natarajan, K., and Janke, C. (2017). The Tubulin Code at a Glance. J. Cel Sci 130 (8), 1347-1353. doi:10.1242/jcs.199471

Gartz Hanson, M., Aiken, J., Sietsema, D. V., Sept, D., Bates, E. A., Niswander, L., et al. (2016). Novel $\alpha$-tubulin Mutation Disrupts Neural Development and Tubulin Proteostasis. Dev. Biol. 409 (2), 406-419. doi:10.1016/ j.ydbio.2015.11.022

Gloster, A., El-Bizri, H., Bamji, S. X., Rogers, D., and Miller, F. D. (1999). Early Induction of T?1 ?-tubulin Transcription in Neurons of the Developing Nervous System. J. Comp. Neurol. 405 (1), 45-60. doi:10.1002/(sici)10969861(19990301)405:1<45:aid-cne4>3.0.co;2-m

Gloster, A., Wu, W., Speelman, A., Weiss, S., Causing, C., Pozniak, C., et al. (1994). The T Alpha 1 Alpha-Tubulin Promoter Specifies Gene Expression as a Function of Neuronal Growth and Regeneration in Transgenic Mice. J. Neurosci. 14 (12), 7319-7330. doi:10.1523/jneurosci.14-12-07319.1994

Gonzalez-Billault, C., Avila, J., and Cáceres, A. (2001). Evidence for the Role of MAP1B in Axon Formation. MBoC 12 (7), 2087-2098. doi:10.1091/ mbc.12.7.2087 
González-Billault, C., Engelke, M., Jimȳnez-Mateos, E. M., Wandosell, F., Cáceres, A., and Avila, J. (2002). Participation of Structural Microtubule-Associated Proteins (MAPs) in the Development of Neuronal Polarity. J. Neurosci. Res. 67 (6), 713-719.

Gonzalez-Garay, M. L., and Cabral, F. (1996). Alpha-Tubulin Limits its Own Synthesis: Evidence for a Mechanism Involving Translational Repression. J. Cel Biol 135 (6 Pt 1), 1525-1534. doi:10.1083/jcb.135.6.1525

Heilemann, M., van de Linde, S., Schüttpelz, M., Kasper, R., Seefeldt, B., Mukherjee, A., et al. (2008). Subdiffraction-resolution Fluorescence Imaging with Conventional Fluorescent Probes. Angew. Chem. Int. Ed. 47 (33), 6172-6176. doi:10.1002/anie.200802376

Hoogendoorn, E., Crosby, K. C., Leyton-Puig, D., Breedijk, R. M. P., Jalink, K., Gadella, T. W. J., et al. (2014). The Fidelity of Stochastic Single-Molecule Superresolution Reconstructions Critically Depends upon Robust Background Estimation. Sci. Rep. 4, 3854. doi:10.1038/srep03854

Hoogenraad, C. C., and Bradke, F. (2009). Control of Neuronal Polarity and Plasticity - a Renaissance for Microtubules? Trends Cel Biol. 19 (12), 669-676. doi:10.1016/j.tcb.2009.08.006

Hotta, T., Fujita, S., Uchimura, S., Noguchi, M., Demura, T., Muto, E., et al. (2016). Affinity Purification and Characterization of Functional Tubulin from Cell Suspension Cultures of Arabidopsis and Tobacco. Plant Physiol. 170 (3), 1189-1205. doi:10.1104/pp.15.01173

Igarashi, M. (2014). Proteomic Identification of the Molecular Basis of Mammalian CNS Growth Cones. Neurosci. Res. 88, 1-15. doi:10.1016/j.neures.2014.07.005

Janke, C., and Kneussel, M. (2010). Tubulin post-translational Modifications: Encoding Functions on the Neuronal Microtubule Cytoskeleton. Trends Neurosciences 33 (8), 362-372. doi:10.1016/j.tins.2010.05.001

Johnson, V., Ayaz, P., Huddleston, P., and Rice, L. M. (2011). Design, Overexpression, and Purification of Polymerization-Blocked Yeast $\alpha \beta-$ Tubulin Mutants. Biochemistry 50 (40), 8636-8644. doi:10.1021/bi2005174

Kapitein, L. C., and Hoogenraad, C. C. (2015). Building the Neuronal Microtubule Cytoskeleton. Neuron 87 (3), 492-506. doi:10.1016/j.neuron.2015.05.046

Keays, D. A., Cleak, J., Huang, G.-J., Edwards, A., Braun, A., Treiber, C. D., et al. (2010). The Role of Tubala in Adult Hippocampal Neurogenesis and the Formation of the Dentate Gyrus. Dev. Neurosci. 32 (4), 268-277. doi:10.1159/ 000319663

Keays, D. A., Tian, G., Poirier, K., Huang, G.-J., Siebold, C., Cleak, J., et al. (2007). Mutations in $\alpha$-Tubulin Cause Abnormal Neuronal Migration in Mice and Lissencephaly in Humans. Cell 128 (1), 45-57. doi:10.1016/j.cell.2006.12.017

Khodiyar, V. K., Maltais, L. J., Sneddon, K. M. B., Smith, J. R., Shimoyama, M., Cabral, F., et al. (2007). A Revised Nomenclature for the Human and Rodent $\alpha$ tubulin Gene Family. Genomics 90 (2), 285-289. doi:10.1016/ j.ygeno.2007.04.008

Kimble, M., Kuzmiak, C., McGovern, K. N., and de Hostos, E. L. (2000). Microtubule Organization and the Effects of GFP-Tubulin Expression inDictyostelium Discoideum. Cell Motil. Cytoskeleton 47 (1), 48-62. doi:10.1002/1097-0169(200009)47:1<48:aid-cm5>3.0.co;2-q

Krol, A., and Feng, G. (2018). Windows of Opportunity: Timing in Neurodevelopmental Disorders. Curr. Opin. Neurobiol. 48, 59-63. doi:10.1016/j.conb.2017.10.014

Kumar, R. A., Pilz, D. T., Babatz, T. D., Cushion, T. D., Harvey, K., Topf, M., et al. (2010). TUBA1A Mutations Cause Wide Spectrum Lissencephaly (Smooth Brain) and Suggest that Multiple Neuronal Migration Pathways Converge on Alpha Tubulins. Hum. Mol. Genet. 19 (14), 2817-2827. doi:10.1093/hmg/ ddq182

Lacroix, B., van Dijk, J., Gold, N. D., Guizetti, J., Aldrian-Herrada, G., Rogowski, K., et al. (2010). Tubulin Polyglutamylation Stimulates Spastin-Mediated Microtubule Severing. J. Cel Biol 189 (6), 945-954. doi:10.1083/jcb.201001024

Leca, I., Phillips, A. W., Hofer, I., Landler, L., Ushakova, L., Cushion, T. D., et al. (2020). A Proteomic Survey of Microtubule-Associated Proteins in a R402H TUBA1A Mutant Mouse. Plos Genet. 16 (11), e1009104. doi:10.1371/ journal.pgen.1009104

Lecourtois, M., Poirier, K., Friocourt, G., Jaglin, X., Goldenberg, A., Saugier-Veber, P., et al. (2010). Human Lissencephaly with Cerebellar Hypoplasia Due to Mutations in TUBA1A: Expansion of the Foetal Neuropathological Phenotype. Acta Neuropathol. 119 (6), 779-789. doi:10.1007/s00401-010-0684-Z

Leung, K.-M., Lu, B., Wong, H. H.-W., Lin, J. Q., Turner-Bridger, B., and Holt, C. E. (2018). Cue-Polarized Transport of $\beta$-actin mRNA Depends on $3^{\prime} U T R$ and
Microtubules in Live Growth Cones. Front. Cel. Neurosci. 12, 300. doi:10.3389/ fncel.2018.00300

Lewis, S., Tian, G., and Cowan, N. (1997). The $\alpha$ - and $\beta$-tubulin Folding Pathways. Trends Cel Biol. 7 (12), 479-484. doi:10.1016/s0962-8924(97)01168-9

Lindwall, C., Fothergill, T., and Richards, L. J. (2007). Commissure Formation in the Mammalian Forebrain. Curr. Opin. Neurobiol. 17 (1), 3-14. doi:10.1016/ j.conb.2007.01.008

Liu, G., and Dwyer, T. (2014). Microtubule Dynamics in Axon Guidance. Neurosci. Bull. 30 (4), 569-583. doi:10.1007/s12264-014-1444-6

Liu, J. S., Schubert, C. R., Fu, X., Fourniol, F. J., Jaiswal, J. K., Houdusse, A., et al. (2012). Molecular Basis for Specific Regulation of Neuronal Kinesin-3 Motors by Doublecortin Family Proteins. Mol. Cel 47 (5), 707-721. doi:10.1016/ j.molcel.2012.06.025

Mack, T. G. A., Koester, M. P., and Pollerberg, G. E. (2000). The MicrotubuleAssociated Protein MAP1B Is Involved in Local Stabilization of Turning Growth Cones. Mol. Cell Neurosci. 15 (1), 51-65. doi:10.1006/mcne.1999.0802

Mansfield, S. G., and Gordon-Weeks, P. R. (1991). Dynamic post-translational Modification of Tubulin in Rat Cerebral Cortical Neurons Extending Neurites in Culture: Effects of Taxol. J. Neurocytol 20 (8), 654-666. doi:10.1007/ bf01187067

Meixner, A., Haverkamp, S., Wässle, H., Führer, S., Thalhammer, J., Kropf, N., et al. (2000). MAP1B Is Required for Axon Guidance and Is Involved in the Development of the central and Peripheral Nervous System. J. Cel Biol 151 (6), 1169-1178. doi:10.1083/jcb.151.6.1169

Miller, F. D., Naus, C. C., Durand, M., Bloom, F. E., and Milner, R. J. (1987). Isotypes of Alpha-Tubulin Are Differentially Regulated during Neuronal Maturation. J. Cel Biol 105 (6 Pt 2), 3065-3073. doi:10.1083/ jcb.105.6.3065

Miller, K. E., and Suter, D. M. (2018). An Integrated Cytoskeletal Model of Neurite Outgrowth. Front. Cel. Neurosci. 12, 447. doi:10.3389/fncel.2018.00447

Nishikimi, M., Oishi, K., and Nakajima, K. (2013). Axon Guidance Mechanisms for Establishment of Callosal Connections. Neural Plast. 2013, 149060. doi:10.1155/2013/149060

Noiges, R., Eichinger, R., Kutschera, W., Fischer, I., Németh, Z., Wiche, G., et al. (2002). Microtubule-associated Protein 1A (MAP1A) and MAP1B: Light Chains Determine Distinct Functional Properties. J. Neurosci. 22 (6), 2106-2114. doi:10.1523/jneurosci.22-06-02106.2002

Oegema, R., Cushion, T. D., Phelps, I. G., Chung, S.-K., Dempsey, J. C., Collins, S., et al. (2015). Recognizable Cerebellar Dysplasia Associated with Mutations in Multiple Tubulin Genes. Hum. Mol. Genet. 24 (18), 5313-5325. doi:10.1093/ $\mathrm{hmg} / \mathrm{ddv} 250$

Ōmura, S., and Crump, A. (2019). Lactacystin: First-In-Class Proteasome Inhibitor Still Excelling and an Exemplar for Future Antibiotic Research. J. Antibiot. (Tokyo) 72 (4), 189-201. doi:10.1038/s41429-019-0141-8

Ovesný, M. (2014). ThunderSTORM: a Comprehensive ImageJ Plug-In for PALM and STORM Data Analysis and Super-resolution Imaging. Bioinformatics 30 (16), 2389-2390.

Palazzo, A., Ackerman, B., and Gundersen, G. G. (2003). Tubulin Acetylation and Cell Motility. Nature 421 (6920), 230. doi:10.1038/421230a

Pilaz, L.-J., Lennox, A. L., Rouanet, J. P., and Silver, D. L. (2016). Dynamic mRNA Transport and Local Translation in Radial Glial Progenitors of the Developing Brain. Curr. Biol. 26 (24), 3383-3392. doi:10.1016/ j.cub.2016.10.040

Piper, M., Lee, A. C., van Horck, F. P., McNeilly, H., Lu, T. B., Harris, W. A., et al. (2015). Differential Requirement of F-Actin and Microtubule Cytoskeleton in Cue-Induced Local Protein Synthesis in Axonal Growth Cones. Neural Dev. 10, 3. doi:10.1186/s13064-015-0031-0

Poirier, K., Keays, D. A., Francis, F., Saillour, Y., Bahi, N., Manouvrier, S., et al. (2007). Large Spectrum of Lissencephaly and Pachygyria Phenotypes Resulting from De Novo Missense Mutations in Tubulin Alpha 1A (TUBA1A). Hum. Mutat. 28 (11), 1055-1064. doi:10.1002/humu.20572

Probst, M. (1901). Uber den Bau des balkenlosen Grobhirns, sowie uber Mikrogyrie and Heterotypie der grauer Substanz. Arch. F Psychiatr. (34), 709-786. doi:10.1007/bf02680175

Qu, C., Dwyer, T., Shao, Q., Yang, T., Huang, H., and Liu, G. (2013). Direct Binding of TUBB3 with DCC Couples Netrin-1 Signaling to Intracellular Microtubule Dynamics in Axon Outgrowth and Guidance. J. Cel Sci 126 (Pt 14), 3070-3081. doi:10.1242/jcs. 122184 
Reed, N. A., Cai, D., Blasius, T. L., Jih, G. T., Meyhofer, E., Gaertig, J., et al. (2006). Microtubule Acetylation Promotes Kinesin-1 Binding and Transport. Curr. Biol. 16 (21), 2166-2172. doi:10.1016/j.cub.2006.09.014

Robson, S. J., and Burgoyne, R. D. (1989). Differential Localisation of Tyrosinated, Detyrosinated, and Acetylated ?-tubulins in Neurites and Growth Cones of Dorsal Root Ganglion Neurons. Cel Motil. Cytoskeleton 12 (4), 273-282. doi: $10.1002 / \mathrm{cm} .970120408$

Rochlin, M. W., Dailey, M. E., and Bridgman, P. C. (1999). Polymerizing Microtubules Activate Site-Directed F-Actin Assembly in Nerve Growth Cones. MBoC 10 (7), 2309-2327. doi:10.1091/mbc.10.7.2309

Roostalu, J., Thomas, C., Cade, N. I., Kunzelmann, S., Taylor, I. A., and Surrey, T. (2020). The Speed of GTP Hydrolysis Determines GTP Cap Size and Controls Microtubule Stability. Elife 9. doi:10.7554/eLife.51992

Rossi, A. M., Fernandes, V. M., and Desplan, C. (2017). Timing Temporal Transitions during Brain Development. Curr. Opin. Neurobiol. 42, 84-92. doi:10.1016/j.conb.2016.11.010

Sakakibara, A., Ando, R., Sapir, T., and Tanaka, T. (2013). Microtubule Dynamics in Neuronal Morphogenesis. Open Biol. 3 (7), 130061. doi:10.1098/rsob.130061

Schatz, P. J., Georges, G. E., Solomon, F., and Botstein, D. (1987). Insertions of up to 17 Amino Acids into a Region of Alpha-Tubulin Do Not Disrupt Function In Vivo. Mol. Cel Biol 7 (10), 3799-3805. doi:10.1128/mcb.7.10.3799-3805.1987

Schneider, C. A., Rasband, W. S., and Eliceiri, K. W. (2012). NIH Image to ImageJ: 25 Years of Image Analysis. Nat. Methods 9 (7), 671-675. doi:10.1038/ nmeth.2089

Schulze, E., Asai, D. J., Bulinski, J. C., and Kirschner, M. (1987). Posttranslational Modification and Microtubule Stability. J. Cel Biol 105 (5), 2167-2177. doi:10.1083/jcb.105.5.2167

Schüttpelz, M. (2010). dSTORM: Real-Time Subdiffraction-Resolution Fluorescence Imaging with Organic Fluorophores, 7571. Bellingham, WA: SPIE BiOS.

Serafini, T., Colamarino, S. A., Leonardo, E. D., Wang, H., Beddington, R., Skarnes, W. C., et al. (1996). Netrin-1 Is Required for Commissural Axon Guidance in the Developing Vertebrate Nervous System. Cell 87 (6), 1001-1014. doi:10.1016/s0092-8674(00)81795-x

Sirajuddin, M., Rice, L. M., and Vale, R. D. (2014). Regulation of Microtubule Motors by Tubulin Isotypes and post-translational Modifications. Nat. Cel Biol 16 (4), 335-344. doi:10.1038/ncb2920

Slater, P. G., Cammarata, G. M., Samuelson, A. G., Magee, A., Hu, Y., and Lowery, L. A. (2019). XMAP215 Promotes Microtubule-F-Actin Interactions to Regulate Growth Cone Microtubules during Axon Guidance in Xenopus laevis. J. Cel Sci 132 (9), jcs224311. doi:10.1242/jcs.224311

Szikora, S., Földi, I., Tóth, K., Migh, E., Vig, A., Bugyi, B., et al. (2017). The Formin DAAM Is Required for Coordination of the Actin and Microtubule Cytoskeleton in Axonal Growth Cones. J. Cel Sci 130 (15), 2506-2519. doi: $10.1242 /$ jcs. 203455

Tian, G., Jaglin, X. H., Keays, D. A., Francis, F., Chelly, J., and Cowan, N. J. (2010). Disease-associated Mutations in TUBA1A Result in a Spectrum of Defects in the Tubulin Folding and Heterodimer Assembly Pathway. Hum. Mol. Genet. 19 (18), 3599-3613. doi:10.1093/hmg/ddq276

Tian, G., Kong, X.-P., Jaglin, X. H., Chelly, J., Keays, D., and Cowan, N. J. (2008). A Pachygyria-Causing $\alpha$-Tubulin Mutation Results in Inefficient Cycling with
CCT and a Deficient Interaction with TBCB. MBoC 19 (3), 1152-1161. doi:10.1091/mbc.e07-09-0861

Tischfield, M. A., Cederquist, G. Y., Gupta, M. L., and Engle, E. C. (2011). Phenotypic Spectrum of the Tubulin-Related Disorders and Functional Implications of Disease-Causing Mutations. Curr. Opin. Genet. Dev. 21 (3), 286-294. doi:10.1016/j.gde.2011.01.003

Tomoda, H., and Omura, S. (2000). Lactacystin, a Proteasome Inhibitor : Discovery and its Application in Cell Biology. Yakugaku Zasshi 120 (10), 935-949. doi:10.1248/yakushi1947.120.10_935

Tymanskyj, S. R., Scales, T. M. E., and Gordon-Weeks, P. R. (2012). MAP1B Enhances Microtubule Assembly Rates and Axon Extension Rates in Developing Neurons. Mol. Cell Neurosci. 49 (2), 110-119. doi:10.1016/ j.men.2011.10.003

Vallee, R. B. (1982). A Taxol-dependent Procedure for the Isolation of Microtubules and Microtubule-Associated Proteins (MAPs). J. Cel Biol 92 (2), 435-442. doi:10.1083/jcb.92.2.435

Wang, Y., Schnitzbauer, J., Hu, Z., Li, X., Cheng, Y., Huang, Z.-L., et al. (2014). Localization Events-Based Sample Drift Correction for Localization Microscopy with Redundant Cross-Correlation Algorithm. Opt. Express 22 (13), 15982-15991. doi:10.1364/oe.22.015982

Welshhans, K., and Bassell, G. J. (2011). Netrin-1-Induced Local -Actin Synthesis and Growth Cone Guidance Requires Zipcode Binding Protein 1. J. Neurosci. 31 (27), 9800-9813. doi:10.1523/jneurosci.0166-11.2011

Zhang, Y., Chen, K., Sloan, S. A., Bennett, M. L., Scholze, A. R., O'Keeffe, S., et al. (2014). An RNA-Sequencing Transcriptome and Splicing Database of Glia, Neurons, and Vascular Cells of the Cerebral Cortex. J. Neurosci. 34 (36), 11929-11947. doi:10.1523/jneurosci.1860-14.2014

Zhang, Y., Sloan, S. A., Clarke, L. E., Caneda, C., Plaza, C. A., Blumenthal, P. D., et al. (2016). Purification and Characterization of Progenitor and Mature Human Astrocytes Reveals Transcriptional and Functional Differences with Mouse. Neuron 89 (1), 37-53. doi:10.1016/j.neuron.2015.11.013

Zieve, G., and Solomon, F. (1984). Direct Isolation of Neuronal Microtubule Skeletons. Mol. Cel Biol 4 (2), 371-374. doi:10.1128/mcb.4.2.371-374.1984

Conflict of Interest: The authors declare that the research was conducted in the absence of any commercial or financial relationships that could be construed as a potential conflict of interest.

Publisher's Note: All claims expressed in this article are solely those of the authors and do not necessarily represent those of their affiliated organizations, or those of the publisher, the editors and the reviewers. Any product that may be evaluated in this article, or claim that may be made by its manufacturer, is not guaranteed or endorsed by the publisher.

Copyright (c) 2022 Buscaglia, Northington, Aiken, Hoff and Bates. This is an openaccess article distributed under the terms of the Creative Commons Attribution License (CC BY). The use, distribution or reproduction in other forums is permitted, provided the original author(s) and the copyright owner(s) are credited and that the original publication in this journal is cited, in accordance with accepted academic practice. No use, distribution or reproduction is permitted which does not comply with these terms. 